TRANSACTIONS OF THE

AMERICAN MATHEMATICAL SOCIETY

Volume 351, Number 1, January 1999, Pages 79-108

S 0002-9947(99)02116-9

\title{
MAXIMAL FUNCTION ESTIMATES OF SOLUTIONS TO GENERAL DISPERSIVE PARTIAL DIFFERENTIAL EQUATIONS
}

\author{
HANS P. HEINIG AND SICHUN WANG
}

\begin{abstract}
Let $u(x, t)=\left(S_{\Omega} f\right)(x, t)$ be the solution of the general dispersive initial value problem:

$$
\partial_{t} u-i \Omega(D) u=0, \quad u(x, 0)=f(x), \quad(x, t) \in \mathbb{R}^{n} \times \mathbb{R}
$$

and $S_{\Omega}^{* *} f$ the global maximal operator of $S_{\Omega} f$. Sharp weighted $L^{p}$-esimates for $S_{\Omega}^{* *} f$ with $f \in H_{s}\left(\mathbb{R}^{n}\right)$ are given for general phase functions $\Omega$.
\end{abstract}

\section{INTRODUCTION}

The initial value problem (IVP) for the free Schrödinger equation

$$
\begin{aligned}
\partial_{t} u+i \Delta_{x} u & =0, \quad(x, t) & \in \mathbb{R}^{n} \times \mathbb{R}, \\
u(x, 0) & =f(x), \quad x & \in \mathbb{R}^{n},
\end{aligned}
$$

has the formal solution

$$
u(x, t)=(S f)(x, t)=(2 \pi)^{-n} \int_{\mathbb{R}^{n}} e^{i x \cdot \xi} e^{i t|\xi|^{2}} \hat{f}(\xi) d \xi,
$$

where

$$
\hat{f}(\xi)=\int_{\mathbb{R}^{n}} e^{-i \xi \cdot x} f(x) d x, \quad \xi \in \mathbb{R}^{n},
$$

is the Fourier trnasform of the initial data $f$ of (1.1).

In order to obtain sufficient and (almost) necessary conditions for the almost everywhere convergence of the solution $u(x, t)$ to the initial data, L. Carleson ([5]) posed the question of finding minimal regularity required of $f$ such that the local maximal operator

$$
\left(S^{*} f\right)(x)=\sup _{|t| \leq 1}|(S f)(x, t)|
$$

is locally integrable.

This problem is completely solved in the one-dimensional case (of the spatial variable) (cf. [11], [12]), however, in the higher dimensional case, the problem is still largely open, although a considerable number of partial results are available ([3], [4], [19], [28]).

Received by the editors May 5, 1996 and, in revised form, July 1, 1996.

1991 Mathematics Subject Classification. Primary 42B25; Secondary 42A45.

Key words and phrases. Dispersive PDE, free Schrödinger equation, phase functions, polynomials of principal type, regular zeroes, weighted $L^{p}$-spaces, Sobolev spaces.

The research of the first author was supported in part by NSERC grant A-4837.

(C)1999 American Mathematical Society 
A natural generalization of Carleson's problem is to replace in (1.1) the Laplacian $\Delta$ by a general differential operator of constant coefficients in the spatial variables and then find minimal regularity for the associated local (and global) maximal function (cf. (1.2)). This problem was studied in a number of papers by various authors, but specifically by C. E. Kenig, G. Ponce and L. Vega ([12]).

Let $D=-i \nabla=-i\left(\partial_{x_{1}}, \partial_{x_{2}}, \ldots, \partial_{x_{n}}\right)$, then the general dispersive initial value problem (GDIVP)

$$
\begin{gathered}
\partial_{t} u-i \Omega(D) u=0, \quad(x, t) \in \mathbb{R}^{n} \times \mathbb{R}, \\
u(x, 0)=f(x), \quad x \in \mathbb{R}^{n}
\end{gathered}
$$

has solution

$$
u(x, t)=\left(S_{\Omega} f\right)(x, t)=(2 \pi)^{-n} \int_{\mathbb{R}^{n}} e^{i x \cdot \xi} e^{i t \Omega(\xi)} \hat{f}(\xi) d \xi
$$

Here $\hat{f}$ is again the Fourier transform of the initial data $f, \Omega$ a smooth phase function and

$$
\Omega(D) u(x)=(2 \pi)^{-n} \int_{\mathbb{R}^{n}} e^{i x \cdot \xi} \Omega(\xi) \hat{u}(\xi) d \xi .
$$

For a given phase function $\Omega$, we define the local and global maximal operators $S_{\Omega}^{*}, S_{\Omega}^{* *}$ by

$$
\left(S_{\Omega}^{*} f\right)(x)=\sup _{0<t<1}\left|\left(S_{\Omega} f\right)(x, t)\right|, \quad f \in \mathcal{S}\left(\mathbb{R}^{n}\right)
$$

respectively

$$
\left(S_{\Omega}^{* *} f\right)(x)=\sup _{t \in \mathbb{R}}\left|\left(S_{\Omega} f\right)(x, t)\right|, \quad f \in \mathcal{S}(\mathbb{R})^{n} .
$$

In this paper we establish sharp images in terms of weight functions $w$ and indices $p$ and $s$, such that, for general smooth phase functions $\Omega$ the inequality

$$
\left\{\int_{\mathbb{R}^{n}}\left|\left(S_{\Omega}^{* *} f\right)(x)\right|^{p} w(x) d x\right\}^{1 / p} \leq C\|f\|_{H_{s}}
$$

holds. Here $C>0$ is a constant independent of $f$,

$$
H_{s}\left(\mathbb{R}^{n}\right)=\left\{f \in \mathcal{S}^{\prime}\left(\mathbb{R}^{n}\right):\|f\|_{H_{s}}<\infty\right\},
$$

where

$$
\|f\|_{H_{s}}=\left\{\int_{\mathbb{R}^{n}}\left(1+|\xi|^{2}\right)^{s}|\hat{f}(\xi)|^{2} d \xi\right\}^{1 / 2}
$$

is the usual norm of the Sobolev spaces $H_{s}\left(\mathbb{R}^{n}\right)$, and $\mathcal{S}^{\prime}\left(\mathbb{R}^{n}\right)$ the dual space of the Schwartz class $\mathcal{S}\left(\mathbb{R}^{n}\right)([10],[25])$.

In particular we consider estimates (1.5), primarily with $p=2$ and $w(x)=$ $|x|^{-a}(1+|x|)^{-b}, a \geq 0, b \geq 0$, for a number of general phase functions $\Omega$. In case $\Omega$ is a polynomial of principal type we obtain sharp estimates in terms of $a$ and $s$ for $p=2$ (Theorem 3.3). If $\Omega$ is a real homogeneous polynomial such that $|\nabla \Omega|$ has finitely many regular zeroes on $S^{n-1}$, the unit sphere in $\mathbb{R}^{n}$, then again sharp estimates with $p \geq 2$ are proved (Theorem 3.4, Proposition 3.2). These main results complement the work of Kenig, Ponce and Vega (cf. [12, Thm. 4.1]) and are given in Section 3.

In Section 4 we illustrate the results by providing various specific phase functions to which our results are applicable. For example in Proposition 4.1, 4, the phase 
function $\Omega$ given by $\Omega(\xi)\left(\sum_{i=1}^{n-1} \xi_{i}^{2}\right)^{m} \xi_{n}, m \geq 1$, an integer, is such that $|\nabla \Omega|^{2}$ is not elliptic, yet a sharp estimate of the form (1.5) holds.

Of course, if $\Omega(\xi)=|\xi|^{2}$ or $\Omega(\xi)=|\xi|^{3}$, with $n=1$, then the GDIVP (1.3) reduces to the free Schrödinger equation, respectively, to the linear version of the $\mathrm{KdV}$ equation

$$
\begin{aligned}
\frac{\partial u}{\partial t}+\frac{\partial^{3} u}{\partial x^{3}} & =0, \quad(x, t) \in \mathbb{R} \times \mathbb{R} \\
u(x, 0) & =f(x)
\end{aligned}
$$

Hence the case $\Omega(\xi)=|\xi|^{d}, d>0$, is of special interest and sharp indices for the global maximal operator, denoted by $S_{d}^{* *}$ in this case, are proved. These results are contained in Section 4 and extend and complement those given in [4], [12], [20], [21], [22].

We begin in the next section with a number of technical results needed in the sequel, some of which may be of independent interest.

As is usual, inequalities (such as (1.5)) are interpreted in the sense that if the right side is finite, so is the left side, and the inequality holds. Also, we write $P \cong Q$, if $P / Q$ is bounded above and below by positive constants, and if $E$ is a set (in $\mathbb{R}^{n}$ ), then $E^{c}$ denotes the complement of $E,|E|$ its Lebesgue measure and $\chi_{E}$ is the characteristic function of $E$. Other notation are introduced as they occur.

\section{Preliminary Results}

Now a number of preliminary results are given which are required in the sequel. The first is a weighted Fourier inequality involving radial weight functions on $\mathbb{R}^{n}$, $n>1$.

Lemma 2.1. Let $a \geq 0, b \geq 0, s_{0} \geq 0$ and $2 \leq p<+\infty$. There is a constant $C>0$ independent of $f$ such that the inequality

$$
\left\{\int_{\mathbb{R}^{n}}|\hat{f}(x)|^{p}|x|^{-a}(1+|x|)^{-b} d x\right\}^{1 / p} \leq C\left\{\int_{\mathbb{R}^{n}}\left(1+|x|^{2}\right)^{s_{0}}|f(x)|^{2} d x\right\}^{1 / 2}
$$

holds, if and only if, $s_{0} \geq n(1 / 2-1 / p)+a / p$ and $a<n$. Moreover, if $\left(1+|x|^{2}\right)^{s_{0}}$ in (2.1) is replaced by $|x|^{2 s_{0}}$ and $b=0$, then (2.1) holds if and only if $s_{0}=n(1 / 2-$ $1 / p)+a / p, a<n$.

Proof. Applying [9, Thm. 3.1] if $p>2$ and [1, Thm. 1] if $p=2$ with $u(x)=$ $|x|^{a / p}(1+|x|)^{-b / p}, v(x)=\left(1+|x|^{2}\right)^{s_{0} / 2}$, then $(2.1)$ is equivalent to

$$
\sup _{s>0}\left(\int_{0}^{s^{-2} K} t^{n-1-a}(1+t)^{-b} d t\right)^{1 / p}\left(\int_{0}^{s^{2} K} t^{n-1}\left(1+t^{2}\right)^{-s_{0}} d t\right)^{1 / 2}<+\infty
$$

where $1 / K$ is the $n$th root of the volume of the unit ball in $\mathbb{R}^{n}$.

Now, if $a \geq n$, the left integral of (2.2) diverges and hence we require $a<n$.

Next, if $s>0$ is large, then

$$
\left(\int_{0}^{s^{-2} K} t^{n-1-a}(1+t)^{-b} d t\right)^{1 / p} \cong C s^{-2(n-a) / p}
$$


and

$$
\left(\int_{0}^{s^{2} K}\left(1+t^{2}\right)^{-s_{0}} t^{n-1} d t\right)^{1 / 2} \cong \begin{cases}C s^{n-2 s_{0}}, & \text { if } n \neq 2 s_{0} \\ \left(C_{1}+C_{2} \ln s\right)^{1 / 2}, & \text { if } n=2 s_{0}\end{cases}
$$

where $C, C_{1}, C_{2}$, are some positive constants. Hence if the supremum (2.2) is finite, then for $n \neq 2 s_{0}$,

$$
\limsup _{s \rightarrow \infty} s^{-2(n-a) / p+n-2 s_{0}}<\infty,
$$

which implies $-2(n-a) / p+n-2 s_{0} \leq 0$, that is, $s_{0} \geq n(1 / 2-1 / p)+a / p$, and if $n=2 s_{0}$, then automatically $s_{0}=n / 2 \geq n(1 / 2-1 / p)+a / p$. Thus the necessity part is proved.

To prove sufficiency, assume $s_{0} \geq n(1 / 2-1 / p)+a / p, a<n$ and write for $s>0$

$F(s)=\left(\int_{0}^{s^{-2} K} t^{n-1-a}(1+t)^{-b} d t\right)^{1 / p}, \quad G(s)=\left(\int_{0}^{s^{2} K} t^{n-1}\left(1+t^{2}\right)^{-s_{0}} d t\right)^{1 / 2}$.

In order to show that (2.2) is satisfied it suffices to show (due to the continuity of $F$ and $G$ ) that both

$$
\limsup _{s \rightarrow 0+} F(s) G(s)<+\infty, \quad \limsup _{s \rightarrow+\infty} F(s) G(s)<+\infty .
$$

But since $s_{0} \geq n(1 / 2-1 / p)+a / p$, the necessity part just proved shows that

$$
\limsup _{s \rightarrow+\infty} F(s) G(s)<+\infty
$$

If $s>0$ is small, then

$$
F(s) \leq \begin{cases}C_{1} s^{-2(n-a-b) / p}, & \text { if } n \neq a+b, \\ C_{2}(\ln (1 / s))^{1 / p}, & \text { if } n=a+b,\end{cases}
$$

and $G(s) \leq C_{3} s^{n}$. It follows that if $n \neq a+b$,

$$
\limsup _{s \rightarrow 0+} F(s) G(s) \leq C \limsup _{s \rightarrow 0+} s^{-2(n-a-b) / p+n}<+\infty,
$$

since $-2(n-a-b) / p+n=2[n(1 / 2-1 / p)+(a+b) / p] \geq 0$, and if $n=a+b$,

$$
\limsup _{s \rightarrow 0+} F(s) G(s) \leq C \limsup _{s \rightarrow 0+}(\ln (1 / s))^{1 / p} s^{n}=0 .
$$

This proves the lemma.

Lemma 2.2 ([25, Ch. 4, Thm. 3.10]). Let $Y$ be a spherical harmonic of order $k$ on the unit $n$-sphere in $\mathbb{R}^{n}$, then

$$
\int_{S^{n-1}} e^{-i s\left(x^{\prime} \cdot \xi^{\prime}\right)} Y\left(\xi^{\prime}\right) d \sigma\left(\xi^{\prime}\right)=(2 \pi)^{n / 2} i^{-k} s^{(2-n) / 2} J_{(n+2 k-2) / 2}(s) Y\left(x^{\prime}\right) .
$$

Here $x^{\prime}, \xi^{\prime} \in S^{n-1}, J_{(n+2 k-2) / 2}(s)$ the Bessel function of order $(n+2 k-2) / 2$ and $d \sigma$ the surface measure on $S^{n-1}$.

Lemma 2.3. (i) Let $n \geq 2, k \geq 0,0<\gamma<n-1$, then there exists a constant $C=C(\gamma, n)>0$, independent of $k$, such that

$$
\int_{0}^{\infty} t^{-\gamma}\left|J_{(n+2 k-2) / 2}(t)\right|^{2} d t \leq C
$$


(ii) If $k \geq 1$, there exists a constant $C>0$, indepedent of $k$ and $t>0$, such that

$$
\left|J_{k / 2}(t)\right| \leq C \text {. }
$$

Proof. It follows from ([32, p. 403]) that

$$
\int_{0}^{\infty} t^{-\gamma}\left|J_{(n+2 k-2) / 2}(t)\right|^{2} d t=C(\gamma) \frac{\Gamma[(k+n / 2)-(\gamma+1) / 2]}{\Gamma[(k+n / 2)+(\gamma-1) / 2]},
$$

where $C(\gamma)=\frac{2^{-\gamma-1} \Gamma(\gamma)}{[\Gamma(1 / 2+\gamma / 2)]^{2}}$. But since for any $a \in \mathbb{R}$,

$$
\Gamma(x+a) \cong \Gamma(x) x^{a}, \quad x \rightarrow \infty,
$$

one can estimate the right side of (2.6) by $C(k+n / 2)^{-\gamma} \leq C$.

(2.5) follows directly from [32, p. 176].

The next two lemmas are Plancherel-type estimates for $\left(S_{\Omega} f\right)(x, \cdot)$, the Fourier transform of $\left(S_{\Omega} f\right)(x, t)$ with respect to $t$, where $\Omega$ are certain phase functions. These technical results are essential in the proof of the main theorems of the next section.

Lemma 2.4. Let $\varphi \in C^{1}(0,+\infty)$ such that $\left|\varphi^{\prime}(t)\right|>0$, and $\Omega(\xi)=\varphi(|\xi|)$. If $0<a<n-1$ and $s_{0} \in \mathbb{R}$, then there exists a constant $C>0$, independent of $f \in \mathcal{S}\left(\mathbb{R}^{n}\right)$, such that

$$
\begin{gathered}
\left\{\int_{\mathbb{R}^{n+1}}\left|\left(S_{\Omega} f\right)^{\wedge}(x, \cdot)(s)\right|^{2}\left(1+s^{2}\right)^{s_{0}}|x|^{-1-a} d s d x\right\}^{1 / 2} \\
\leq C\left\{\int_{\mathbb{R}^{n}} \frac{\left(1+|\varphi(|\xi|)|^{2}\right)^{s_{0}}}{\left|\varphi^{\prime}(|\xi|)\right|}|\xi|^{a}|\hat{f}(\xi)|^{2} d \xi\right\}^{1 / 2}
\end{gathered}
$$

holds.

Proof. We shall prove the result for $\varphi^{\prime}(t)>0$ only, since the argument for the case $\varphi^{\prime}(t)<0$ is identical. Let $g \in \mathcal{S}(\mathbb{R})$, and $A=\lim _{t \rightarrow 0+} \varphi(t), B=\lim _{t \rightarrow+\infty} \varphi(t)$, then an interchange of order of integration, justified by Fubini's theorem, a change to polar coordinates and a change of variables yields

$$
\begin{aligned}
\int_{\mathbb{R}}( & \left.S_{\Omega} f\right)(x, t) \hat{g}(t) d t \\
& =(2 \pi)^{-n} \int_{\mathbb{R}} \int_{\mathbb{R}^{n}}\left(e^{i x \cdot \xi} e^{i t \Omega(\xi)} \hat{f}(\xi) d \xi\right) \hat{g}(t) d t \\
& =(2 \pi)^{-n} \int_{\mathbb{R}^{n}} e^{i x \cdot \xi} \hat{f}(\xi) \int_{\mathbb{R}} e^{i t \varphi(|\xi|)} \hat{g}(t) d t d \xi \\
& =(2 \pi)^{1-n} \int_{0}^{\infty} r^{n-1} g(\varphi(r)) \int_{S^{n-1}} e^{i r|x|\left(x^{\prime} \cdot \xi^{\prime}\right)} \hat{f}\left(r \xi^{\prime}\right) d \sigma\left(\xi^{\prime}\right) d r \\
& =(2 \pi)^{1-n} \int_{A}^{B} g(s) \frac{\left(\varphi^{-1}(s)\right)^{n-1}}{\varphi^{\prime}\left(\varphi^{-1}(s)\right)} \int_{S^{n-1}} e^{i \varphi^{-1}(s)|x|\left(x^{\prime} \cdot \xi^{\prime}\right)} \hat{f}\left(\varphi^{-1}(s) \xi^{\prime}\right) d \sigma\left(\xi^{\prime}\right) d s .
\end{aligned}
$$

Hence $\left(S_{\Omega} f\right) \uparrow(x, \cdot)(s)$ exists as a tempered distribution and is equal to

$$
\begin{aligned}
\left(S_{\Omega} f\right) & \curlyvee(x, \cdot)(s) \\
& =(2 \pi)^{1-n} \chi_{(A, B)}(s) \frac{\left(\varphi^{-1}(s)\right)^{n-1}}{\varphi^{\prime}\left(\varphi^{-1}(s)\right)} \int_{S^{n-1}} e^{i \varphi^{-1}(s)|x|\left(x^{\prime} \cdot \xi^{\prime}\right)} \hat{f}\left(\varphi^{-1}(s) \xi^{\prime}\right) d \sigma\left(\xi^{\prime}\right) .
\end{aligned}
$$


Substituting this expression into the left side of (2.7) yields

$$
\begin{aligned}
\int_{\mathbb{R}}(1+ & \left.s^{2}\right)^{s_{0}}\left(\int_{\mathbb{R}^{n}}\left|\left(S_{\Omega} f\right)^{-}(x, \cdot)(s)\right|^{2} \frac{1}{|x|^{1+a}} d x\right) d s \\
= & C \int_{A}^{B}\left(1+s^{2}\right)^{s_{0}} \frac{\left|\varphi^{-1}(s)\right|^{2(n-1)}}{\left|\varphi^{\prime}\left(\varphi^{-1}(s)\right)\right|^{2}} \\
& \times\left(\int_{\mathbb{R}^{n}}\left|\int_{S^{n-1}} e^{-i \varphi^{-1}(s)|x|\left(x^{\prime} \cdot \xi^{\prime}\right)} \hat{f}\left(\varphi^{-1}(s) \xi^{\prime}\right) d \sigma\left(\xi^{\prime}\right)\right|^{2} \frac{1}{|x|^{1+a}} d x\right) d s \\
= & C \int_{0}^{\infty}\left(1+|\varphi(r)|^{2}\right)^{s_{0}} \frac{r^{2(n-1)}}{\left|\varphi^{\prime}(r)\right|^{2}}\left|\varphi^{\prime}(r)\right| \\
& \times\left(\int_{\mathbb{R}^{n}}\left|\int_{S^{n-1}} e^{i r|x|\left(x^{\prime} \cdot \xi^{\prime}\right)} \hat{f}\left(r \xi^{\prime}\right) d \sigma\left(\xi^{\prime}\right)\right|^{2} \frac{1}{|x|^{1+a}} d x\right) d r,
\end{aligned}
$$

where the formal calculations are clearly justified. Next, we estimate the inner integrals. Let $H_{n}^{(k)}$ denote the vector space of spherical harmonics of order $k$ on $S^{n-1}$, then $\left(\left[25\right.\right.$, Ch. 4]) $H_{n}^{(k)}$ is finite-dimensional with dimension say, $d_{n, k}$. If $\left\{Y_{k, l}\right\}_{l=1}^{d_{n, k}}$ denotes a real-valued orthonormal basis of $H_{n}^{(k)}$, then as in [25, Ch. 4] $\bigcup_{k=0}^{\infty}\left\{Y_{k, l}\right\}_{l=1}^{d_{n, k}}$ is a complete orthonormal basis of $L^{2}\left(S^{n-1}\right)$.

Let

$$
a_{k, l}(r)=\int_{S^{n-1}} Y_{k, l}\left(\xi^{\prime}\right) \hat{f}\left(r \xi^{\prime}\right) d \sigma\left(\xi^{\prime}\right)
$$

then by Parseval's formula

$$
\langle a, b\rangle=\sum_{k, l}\left\langle a, Y_{k, l}\right\rangle \overline{\left\langle b, Y_{k, l}\right\rangle}
$$

with $a=\hat{f}\left(r \xi^{\prime}\right), b=e^{-i r|x|\left(x^{\prime} \cdot \xi^{\prime}\right)}$, and we obtain

$$
\begin{aligned}
\int_{S^{n-1}} & e^{i r|x|\left(x^{\prime} \cdot \xi^{\prime}\right)} \hat{f}\left(r \xi^{\prime}\right) d \sigma\left(\xi^{\prime}\right) \\
= & \sum_{k, l}\left(\int_{S^{n-1}} \hat{f}\left(r \xi^{\prime}\right) Y_{k, l}\left(\xi^{\prime}\right) d \sigma\left(\xi^{\prime}\right)\right)\left(\int_{S^{n-1}} e^{i r|x|\left(x^{\prime} \cdot \xi^{\prime}\right)} Y_{k, l}\left(\xi^{\prime}\right) d \sigma\left(\xi^{\prime}\right)\right) \\
= & \sum_{k, l} a_{k, l}(r)\left(\int_{S^{n-1}} e^{i r|x|\left(x^{\prime} \cdot \xi^{\prime}\right)} Y_{k, l}\left(\xi^{\prime}\right) d \sigma\left(\xi^{\prime}\right)\right)
\end{aligned}
$$

But by Lemma 2.2, this is equal to

$$
\begin{aligned}
(2 \pi)^{n / 2} & \sum_{k=0}^{\infty} \sum_{l=1}^{d_{n, k}} i^{-k}(r|x|)^{1-n / 2} J_{(n+2 k-2) / 2}(r|x|) a_{k, l}(r) Y_{k, l}\left(-x^{\prime}\right) \\
= & (2 \pi)^{n / 2}(r|x|)^{1-n / 2} \sum_{k=0}^{\infty} \sum_{l=1}^{d_{n, k}} i^{-k} a_{k, l}(r) J_{(n+2 k-2) / 2}(r|x|) Y_{k, l}\left(-x^{\prime}\right)
\end{aligned}
$$


Hence a change to polar coordinates shows that

$$
\begin{aligned}
& \int_{\mathbb{R}^{n}}\left|\int_{S^{n-1}} e^{i r|x|\left(x^{\prime} \cdot \xi^{\prime}\right)} \hat{f}\left(r \xi^{\prime}\right) d \sigma\left(\xi^{\prime}\right)\right|^{2} \frac{1}{|x|^{1+a}} d x \\
&=C \int_{0}^{\infty} s^{n-1} s^{-1-a}(r s)^{2-n} \\
& \times {\left[\int_{S^{n-1}}\left|\sum_{k=0}^{\infty} \sum_{l=1}^{d_{n, k}} i^{-k} a_{k, l}(r) J_{(n+2 k-2) / 2}(r s) Y_{k, l}\left(-x^{\prime}\right)\right|^{2} d \sigma\left(x^{\prime}\right)\right] d s } \\
&= C \int_{0}^{\infty} r^{2-n} s^{-a} \\
& \times {\left[\int_{S^{n-1}}\left|\sum_{k=0}^{\infty} \sum_{l=1}^{d_{n, k}} i^{-k} a_{k, l}(r) J_{(n+2 k-2) / 2}(r s) Y_{k, l}\left(-x^{\prime}\right)\right|^{2} d \sigma\left(x^{\prime}\right)\right] d s } \\
&=\left.C r^{2-n} \int_{0}^{\infty} s^{-a}\right] \\
& \times {\left[\int_{S^{n-1}}\left|\sum_{k=0}^{\infty} \sum_{l=1}^{d_{n, k}} i^{-k} a_{k, l}(r) J_{(n+2 k-2) / 2}(r s) Y_{k, l}\left(-x^{\prime}\right)\right|^{2} d \sigma\left(x^{\prime}\right)\right] d s } \\
&= C r^{2-n} \sum_{k=0}^{\infty} \sum_{l=1}^{d_{n, k}}\left(\left|a_{k, l}(r)\right|^{2} \int_{0}^{\infty} s^{-a}\left|J_{(n+2 k-2) / 2}(r s)\right|^{2} d s\right) \\
&= C r^{1+a-n} \sum_{k=0}^{\infty} \sum_{l=1}^{d_{n, k}}\left|a_{k, l}(r)\right|^{2} \int_{0}^{\infty} t^{-a}\left|J_{(n+2 k-2) / 2}(t)\right|^{2} d t \\
&
\end{aligned}
$$

where the last inequality follows from (2.4). Substituting (2.10) into (2.8) and using (2.9), it follows that the left side of (2.7) is dominated by

$$
\begin{gathered}
C\left(\int_{0}^{\infty} \frac{\left(1+|\varphi(r)|^{2}\right)^{s_{0}}}{\left|\varphi^{\prime}(r)\right|} r^{2 n-2} r^{1+a-n}\left(\sum_{k=0}^{\infty} \sum_{l=1}^{d_{n, k}}\left|a_{k, l}\right|^{2}\right) d r\right)^{1 / 2} \\
=C\left(\int_{\mathbb{R}^{n}} \frac{\left(1+|\varphi(|\xi|)|^{2}\right)^{s_{0}}}{\left|\varphi^{\prime}(|\xi|)\right|}|\xi|^{a}|\hat{f}(\xi)|^{2} d \xi\right)^{1 / 2} .
\end{gathered}
$$

The last equality is obtained from Plancherel's theorem

$$
\int_{S^{n-1}}\left|\hat{f}\left(r \xi^{\prime}\right)\right|^{2} d \sigma\left(\xi^{\prime}\right)=\sum_{k=0}^{\infty} \sum_{l=1}^{d_{n, k}}\left|a_{k, l}\right|^{2}=\sum_{k=0}^{\infty} \sum_{l=1}^{d_{n, k}}\left|\int_{S^{n-1}} \hat{f}\left(r \xi^{\prime}\right) Y_{k, l}\left(\xi^{\prime}\right) d \sigma\left(\xi^{\prime}\right)\right|^{2},
$$

and a change from polar coordinates. Thus Lemma 2.4 is proved.

In the sequel $S_{\Omega, D}, D$ an open set in $\mathbb{R}^{n}$, is defined by

$$
\left(S_{\Omega, D} f\right)(x, t)=(2 \pi)^{-n} \int_{D} e^{i x \cdot \xi} e^{i t \Omega(\xi)} \hat{f}(\xi) d \xi
$$


and we write $S_{\Omega, \mathbb{R}^{n}}=S_{\Omega}$. Moreover as in the previous lemma $\left(S_{\Omega, D} f\right)^{\wedge}(x, \cdot)$ denotes the Fourier transform of $\left(S_{\Omega, D} f\right)(x, t)$ in the $t$ variable.

Lemma 2.5. (I) Let $D$ be an open set in $\mathbb{R}^{n}$ and $\Omega$ a real-valued $C^{1}(D)$-function, such that

(i) $\nabla \Omega(\xi) \neq 0$, for all $\xi \in D$;

(ii) There exists a positive integer $N$ and a measurable set $E \subset \mathbb{R}^{n-1}$ with $(n-1)$-dimensional Lebesgue measure equal to zero, such that, for every $\tau$ and every $\bar{\xi}_{k}=\left(\xi_{1}, \ldots, \xi_{k-1}, \xi_{k+1}, \ldots, \xi_{n}\right) \in \mathbb{R}^{n-1} \backslash E, 1 \leq k \leq n$,

$$
\Omega\left(\xi_{1}, \ldots, \xi_{k-1}, x, \xi_{k+1}, \ldots, \xi_{n}\right)=\tau
$$

(as a function of $x$ ) has at most $N$ solutions.

If $\left(S_{\Omega} f\right)^{\wedge}(x, \cdot)(\tau)$ is the Fourier transform of $S_{\Omega} f(x, t)$ with respect to $t$, then for $s_{0} \in \mathbb{R}, 0 \leq a<n-1,0 \leq a_{1}<1,0<b_{1}<+\infty$, with $a_{1}+b_{1}>1$, there exists a constant $C>0$ independent of $f \in \mathcal{S}\left(\mathbb{R}^{n}\right)$, such that

$$
\begin{aligned}
& \left(\int_{\mathbb{R}^{n}} \int_{\mathbb{R}}\left(1+\tau^{2}\right)^{s_{0}}\left|\left(S_{\Omega, D} f\right)^{\wedge}(x, \cdot)(\tau)\right|^{2} \frac{1}{|x|^{a+a_{1}}(1+|x|)^{b_{1}}} d \tau d x\right)^{1 / 2} \\
& \leq C\left(\int_{D} \frac{\left(1+|\Omega|^{2}\right)^{s_{0}}}{|\nabla \Omega|}|\xi|^{a}|\hat{f}(\xi)|^{2} d \xi\right)^{1 / 2} .
\end{aligned}
$$

(II) If $\Omega$ is any non-constant polynomial satisfying (i) (but without the restriction (ii)), then (2.11) holds for $f \in \mathcal{S}\left(\mathbb{R}^{n}\right)$.

Proof. (I) Let $\xi_{0}$ be any fixed point of $D$, then $\nabla \Omega\left(\xi_{0}\right) \neq 0$ and hence there exists an integer $k, 1 \leq k \leq n$, such that

$$
\left|\frac{\partial \Omega}{\partial \xi_{k}}\left(\xi_{0}\right)\right|>\frac{1}{2 n}\left|\nabla \Omega\left(\xi_{0}\right)\right| .
$$

By continuity, there is an open ball $B_{1}\left(\xi_{0}\right)$ centered at $\xi_{0}$ such that for any $\xi \in$ $B_{1}\left(\xi_{0}\right)$

$$
\left|\frac{\partial \Omega}{\partial \xi_{k}}(\xi)\right|>\frac{1}{2 n}|\nabla \Omega(\xi)| .
$$

Now define a mapping $T^{(k)}$ on the ball $B_{1}\left(\xi_{0}\right)$ as follows:

$$
T^{(k)}\left(\xi_{1}, \xi_{2}, \ldots, \xi_{n}\right)=\left(\xi_{1}, \ldots, \xi_{k-1}, \Omega(\xi), \xi_{k+1}, \ldots, \xi_{n}\right),
$$

where $\xi=\left(\xi_{1}, \xi_{2}, \ldots, \xi_{n}\right) \in B_{1}\left(\xi_{0}\right)$. The Jacobian of $T^{(k)}$, denoted by $D T^{(k)}$, is non-singular on $B_{1}\left(\xi_{0}\right)$, since

$$
\left|\operatorname{det}\left(D T^{(k)}(\xi)\right)\right|=\left|\frac{\partial \Omega}{\partial \xi_{k}}(\xi)\right|>\frac{1}{2 n}|\nabla \Omega(\xi)|>0 .
$$

Hence by the Implicit Function Theorem, there exists an open ball $B\left(\xi_{0}\right)$ centered at $\xi_{0}$, such that $\left.T^{(k)}\right|_{B\left(\xi_{0}\right)}$, the restriction of $T^{(k)}$ to $B\left(\xi_{0}\right)$, is a local $C^{1}$ diffeomorphism. Clearly the collection of open balls $\left\{B\left(\xi_{0}\right): \xi_{0} \in D\right\}$ forms an open cover of $D$ and by Lindelöf's theorem there exists a countable subcover $\left\{B_{m}\right\}_{m=1}^{\infty}$ 
of $D$. Let

$$
\begin{aligned}
A_{1} & =B_{1}, \\
A_{2} & =B_{2} \backslash \bar{B}_{1}, \\
& \cdots \\
A_{n} & =B_{n} \backslash\left(\bar{B}_{1} \cup \bar{B}_{2} \cup \cdots \cup \bar{B}_{n-1}\right),
\end{aligned}
$$

where $\bar{B}_{k}$ denotes the closure of $B_{k}$, then $\left\{A_{n}\right\}_{n=1}^{\infty}$ is a sequence of disjoint open subsets of $D$ such that the Lebesgue measure of $D \backslash \bigcup_{n=1}^{\infty} A_{n}$ is zero.

Divide $\left\{A_{n}\right\}_{n=1}^{\infty}$ into $n$ subsequences $\left\{A_{k, l}\right\}_{l=1}^{\infty}, 1 \leq k \leq n$, satisfying the following two conditions:

(i) $\left.T^{(k)}\right|_{A_{k, l}}$, the restriction of $T^{(k)}$ to $A_{k, l}$, is a local diffeomorphism,

(ii) $\forall \xi \in A_{k, l},\left|\frac{\partial \Omega}{\partial \xi_{k}}(\xi)\right|>\frac{1}{2 n}|\nabla \Omega(\xi)|$.

Now let $B_{k, l}=T^{(k)}\left(A_{k, l}\right)$ be the image of $A_{k, l}$ under $T^{(k)}$ and

$$
\left(T^{(k)}\right)^{-1}(\eta)=\left(\eta_{1}, \ldots, \eta_{k-1}, q_{k, l}(\eta), \eta_{k+1}, \ldots, \eta_{n}\right), \quad \eta \in B_{k, l} .
$$

Extend the domain of definition of $q_{k, l}$ to $\mathbb{R}^{n}$ by setting $q_{k, l}(\eta)=\alpha$, if $\eta \in B_{k, l}^{c}$, where $\alpha$ is any fixed real number. Then we obtain a mapping, denoted by $S_{k, l}^{(1)}$, from $\mathbb{R}^{n}$ to $\mathbb{R}^{n}$, that is,

$$
S_{k, l}^{(1)}(\eta)=\left(\eta_{1}, \ldots, \eta_{k-1}, q_{k, l}(\eta), \eta_{k+1}, \ldots, \eta_{n}\right)
$$

Finally, let $\xi^{*}$ be an arbitrary fixed point of $D$ and define another mapping $S_{k, l}^{(2)}$ from $\mathbb{R}^{n}$ to $D$ by

$$
S_{k, l}^{(2)}(\eta)= \begin{cases}\left(\eta_{1}, \ldots, \eta_{k-1}, q_{k, l}(\eta), \eta_{k+1}, \ldots, \eta_{n}\right), & \eta \in B_{k, l} \\ \xi^{*}, & \eta \in B_{k, l}^{c}\end{cases}
$$

Then for any $\varphi \in \mathcal{S}\left(\mathbb{R}^{n}\right)$,

$$
\begin{aligned}
\int_{\mathbb{R}}\left(S_{\Omega, D} f\right)^{\wedge}(x, \cdot)(\tau) \varphi(\tau) d \tau \\
=\int_{\mathbb{R}}\left(S_{\Omega, D} f\right)(x, t) \hat{\varphi}(t) d t \\
=(2 \pi)^{-n} \int_{\mathbb{R}}\left(\int_{D} e^{i x \cdot \xi} e^{i t \Omega(\xi)} \hat{f}(\xi) d \xi\right) \hat{\varphi}(t) d t \\
=(2 \pi)^{1-n} \int_{D} e^{i x \cdot \xi} \hat{f}(\xi) \varphi(\Omega(\xi)) d \xi \\
=(2 \pi)^{1-n} \int_{D} e^{i x \cdot \xi}\left(\sum_{k=1}^{n} \sum_{l=1}^{+\infty} \chi_{A_{k, l}}(\xi)\right) \hat{f}(\xi) \varphi(\Omega(\xi)) d \xi \\
=(2 \pi)^{1-n} \int_{D}\left(\sum_{k=1}^{n} \sum_{l=1}^{+\infty} \chi_{A_{k, l}}(\xi) e^{i x \cdot \xi} \hat{f}(\xi) \varphi(\Omega(\xi))\right) d \xi \\
=(2 \pi)^{1-n} \sum_{k=1}^{n} \sum_{l=1}^{+\infty}\left(\int_{D} \chi_{A_{k, l}}(\xi) e^{i x \cdot \xi} \hat{f}(\xi) \varphi(\Omega(\xi)) d \xi\right),
\end{aligned}
$$


and therefore

$$
\begin{aligned}
\int_{\mathbb{R}}\left(S_{\Omega, D} f\right)^{\wedge}(x, \cdot)(\tau) \varphi(\tau) d \tau \\
\quad=(2 \pi)^{1-n} \sum_{k=1}^{n} \sum_{l=1}^{+\infty}\left(\int_{A_{k, l}} e^{i x \cdot \xi} \hat{f}(\xi) \varphi(\Omega(\xi)) d \xi\right) .
\end{aligned}
$$

Making the change of variables

$$
T^{(k)}\left(\xi_{1}, \xi_{2}, \ldots, \xi_{n}\right)=\left(\eta_{1}, \eta_{2}, \ldots, \eta_{n}\right), \quad \xi \in A_{k, l},
$$

and writing

$$
\begin{aligned}
G_{k, l}(\eta)= & G_{k, l}\left(\bar{\eta}_{k}, \eta_{k}\right)=\hat{f}\left(S_{k, l}^{(2)}\left(\bar{\eta}_{k}, \eta_{k}\right)\right)\left|\frac{\partial \Omega}{\partial \xi_{k}}\left(S_{k, l}^{(2)}\left(\bar{\eta}_{k}, \eta_{k}\right)\right)\right|^{-1} \\
& \left\{\begin{array}{l}
\bar{x}_{k}=\left(x_{1}, \ldots, x_{k-1}, x_{k+1}, \ldots, x_{n}\right) \\
\bar{\eta}_{k}=\left(\eta_{1}, \ldots, \eta_{k-1}, \eta_{k+1}, \ldots, \eta_{n}\right) \\
S_{k, l}^{(2)}\left(\bar{\eta}_{k}, \eta_{k}\right)=S_{k, l}^{(2)}(\eta) \\
q_{k, l}\left(\bar{\eta}_{k}, \eta_{k}\right)=q_{k, l}(\eta)
\end{array}\right.
\end{aligned}
$$

it follows from

$$
\begin{aligned}
\int_{A_{k, l}} & e^{i x \cdot \xi} \hat{f}(\xi) \varphi(\Omega(\xi)) d \xi \\
& =\int_{B_{k, l}} e^{i x \cdot\left(T^{(k)}\right)^{-1}(\eta)} \hat{f}\left(\left(T^{(k)}\right)^{-1}(\eta)\right)\left|\frac{\partial \Omega}{\partial \xi_{k}}\left(\left(T^{(k)}\right)^{-1}(\eta)\right)\right|^{-1} \varphi\left(\eta_{k}\right) d \eta \\
& =\int_{\mathbb{R}^{n}} e^{i x \cdot S_{k, l}^{(1)}(\eta)} \chi_{B_{k, l}}(\eta) \hat{f}\left(S_{k, l}^{(2)}(\eta)\right)\left|\frac{\partial \Omega}{\partial \xi_{k}}\left(S_{k, l}^{(2)}(\eta)\right)\right|^{-1} \varphi\left(\eta_{k}\right) d \eta \\
& =\int_{\mathbb{R}} \varphi\left(\eta_{k}\right) \int_{\mathbb{R}^{n-1}} e^{i\left[x_{k} q_{k, l}\left(\bar{\eta}_{k}, \eta_{k}\right)+\bar{x}_{k} \cdot \bar{\eta}_{k}\right]} \chi_{B_{k, l}}\left(\bar{\eta}_{k}, \eta_{k}\right) G_{k, l}\left(\bar{\eta}_{k}, \eta_{k}\right) d \bar{\eta}_{k} d \eta_{k} \\
& =\int_{\mathbb{R}} \varphi(\tau) \int_{\mathbb{R}^{n-1}} e^{i\left[\bar{x}_{k} \cdot \bar{\eta}_{k}+x_{k} q_{k, l}\left(\bar{\eta}_{k}, \tau\right)\right]} \chi_{B_{k, l}}\left(\bar{\eta}_{k}, \tau\right) G_{k, l}\left(\bar{\eta}_{k}, \tau\right) d \bar{\eta}_{k} d \tau .
\end{aligned}
$$

Therefore by (2.13)

$$
\begin{aligned}
\int_{\mathbb{R}}( & \left.S_{\Omega, D} f\right)^{\wedge}(x, \cdot)(\tau) \varphi(\tau) d \tau \\
= & \sum_{k=1}^{n} \sum_{l=1}^{\infty} \frac{1}{(2 \pi)^{n-1}} \int_{\mathbb{R}} \varphi(\tau) \\
& \times\left[\int_{\mathbb{R}^{n-1}} e^{i\left[\bar{x}_{k} \cdot \bar{\eta}_{k}+x_{k} q_{k, l}\left(\bar{\eta}_{k}, \tau\right)\right]} \chi_{B_{k, l}}\left(\bar{\eta}_{k}, \tau\right) G_{k, l}\left(\bar{\eta}_{k}, \tau\right) d \bar{\eta}_{k}\right] d \tau \\
= & \int_{\mathbb{R}} \varphi(\tau) F(x, \tau) d \tau
\end{aligned}
$$

where

$$
F(x, \tau)=(2 \pi)^{1-n} \sum_{k=1}^{n} \sum_{l=1}^{\infty} \int_{\mathbb{R}^{n-1}} e^{i\left[\bar{x}_{k} \cdot \bar{\eta}_{k}+x_{k} q_{k, l}\left(\bar{\eta}_{k}, \tau\right)\right]} \chi_{B_{k, l}}\left(\bar{\eta}_{k}, \tau\right) G_{k, l}\left(\bar{\eta}_{k}, \tau\right) d \bar{\eta}_{k} .
$$


Here the interchange of integration and summation follows from Fubini's theorem since

$$
\begin{aligned}
\int_{\mathbb{R}} \sum_{k=1}^{n} & \sum_{l=1}^{\infty}|\varphi(\tau)|\left|\int_{\mathbb{R}^{n-1}} e^{i\left[\bar{x}_{k} \cdot \bar{\eta}_{k}+x_{k} q_{k, l}\left(\bar{\eta}_{k}, \tau\right)\right]} \chi_{B_{k, l}}\left(\bar{\eta}_{k}, \tau\right) G_{k, l}\left(\bar{\eta}_{k}, \tau\right) d \bar{\eta}_{k}\right| d \tau \\
\leq & \sum_{k=1}^{n} \sum_{l=1}^{\infty} \int_{\mathbb{R}} \int_{\mathbb{R}^{n-1}}|\varphi(\tau)| \chi_{B_{k, l}}\left(\bar{\eta}_{k}, \tau\right) \\
& \times\left|\frac{\partial \Omega}{\partial \xi_{k}}\left(S_{k, l}^{(2)}\left(\bar{\eta}_{k}, \tau\right)\right)\right|^{-1}\left|\hat{f}\left(S_{k, l}^{(2)}\left(\bar{\eta}_{k}, \tau\right)\right)\right| d \bar{\eta}_{k} d \tau \\
= & \sum_{k=1}^{n} \sum_{l=1}^{\infty} \int_{B_{k, l}} \chi_{B_{k, l}}(\eta)\left|\varphi\left(\eta_{k}\right)\right|\left|\frac{\partial \Omega}{\partial \xi_{k}}\left(S_{k, l}^{(2)}(\eta)\right)\right|^{-1}\left|\hat{f}\left(S_{k, l}^{(2)}(\eta)\right)\right| d \eta \\
= & \sum_{k=1}^{n} \sum_{l=1}^{\infty} \int_{A_{k, l}} \chi_{A_{k, l}}(\xi)|\varphi(\Omega(\xi))||\hat{f}(\xi)| d \xi \\
= & \int_{D}|\hat{f}(\xi)||\varphi(\Omega(\xi))| d \xi<+\infty .
\end{aligned}
$$

But $\varphi \in \mathcal{S}(\mathbb{R})$ is arbitrary and therefore

$$
\begin{aligned}
& \left(S_{\Omega, D} f\right)^{\wedge}(x, \cdot)(\tau)=F(x, \tau) \\
& \quad=(2 \pi)^{1-n} \sum_{k=1}^{n} \sum_{l=1}^{\infty} \int_{\mathbb{R}^{n-1}} e^{i\left[\bar{x}_{k} \cdot \bar{\eta}_{k}+x_{k} q_{k, l}\left(\bar{\eta}_{k}, \tau\right)\right]} \chi_{B_{k, l}}\left(\bar{\eta}_{k}, \tau\right) G_{k, l}\left(\bar{\eta}_{k}, \tau\right) d \bar{\eta}_{k} \\
& \quad=(2 \pi)^{1-n} \sum_{k=1}^{n} \int_{\mathbb{R}^{n-1}} e^{i \bar{x}_{k} \cdot \bar{\eta}_{k}}\left(\sum_{l=1}^{\infty} e^{i x_{k} q_{k, l}\left(\bar{\eta}_{k}, \tau\right)} \chi_{B_{k, l}}\left(\bar{\eta}_{k}, \tau\right) G_{k, l}\left(\bar{\eta}_{k}, \tau\right)\right) d \bar{\eta}_{k},
\end{aligned}
$$

where the interchange of summation and integration here is clearly satisfied.

Write

$$
\begin{aligned}
F_{k}\left(x_{k}, \tau, \bar{\eta}_{k}\right) & =(2 \pi)^{1-n} \sum_{l=1}^{\infty}\left(e^{i x_{k} q_{k, l}\left(\bar{\eta}_{k}, \tau\right)} \chi_{B_{k, l}}\left(\bar{\eta}_{k}, \tau\right) G_{k, l}\left(\bar{\eta}_{k}, \tau\right)\right) \\
& =\sum_{l=1}^{\infty} F_{k, l}\left(x_{k}, \tau, \bar{\eta}_{k}\right)
\end{aligned}
$$

and let $0 \leq a<n-1,0 \leq a_{1}<1,0<b_{1}<+\infty$ with $a_{1}+b_{1}>1, s_{0} \in \mathbb{R}$. 
Then

$$
\begin{aligned}
\int_{\mathbb{R}^{n}} \int_{\mathbb{R}} & \left(1+\tau^{2}\right)^{s_{0}}\left|\left(S_{\Omega, D} f\right)^{\curlyvee}(x, \cdot)(\tau)\right|^{2} \frac{1}{|x|^{a+a_{1}}(1+|x|)^{b_{1}}} d \tau d x \\
= & \int_{\mathbb{R}^{n}} \int_{\mathbb{R}^{2}}\left(1+\tau^{2}\right)^{s_{0}}\left|\sum_{k=1}^{n} \int_{\mathbb{R}^{n-1}} e^{i \bar{x}_{k} \cdot \bar{\eta}_{k}} F_{k}\left(x_{k}, \tau, \bar{\eta}_{k}\right) d \bar{\eta}_{k}\right|^{2} \\
& \times \frac{1}{|x|^{a+a_{1}}(1+|x|)^{b_{1}}} d \tau d x \\
\leq & n \int_{\mathbb{R}^{n}} \int_{\mathbb{R}}\left(1+|\tau|^{2}\right)^{s_{0}} \sum_{k=1}^{n}\left|\int_{\mathbb{R}^{n-1}} e^{i \bar{x}_{k} \cdot \bar{\eta}_{k}} F_{k}\left(x_{k}, \tau, \bar{\eta}_{k}\right) d \bar{\eta}_{k}\right|^{2} \\
& \times \frac{1}{|x|^{a+a_{1}}(1+|x|)^{b_{1}}} d \tau d x \\
= & n \sum_{k=1}^{n} \int_{\mathbb{R}}\left(1+\tau^{2}\right)^{s_{0}} \int_{\mathbb{R}^{n}}\left|\int_{\mathbb{R}^{n-1}} e^{i \bar{x}_{k} \cdot \bar{\eta}_{k}} F_{k}\left(x_{k}, \tau, \bar{\eta}_{k}\right) d \bar{\eta}_{k}\right|^{2} \\
& \times \frac{1}{|x|^{a+a_{1}}(1+|x|)^{b_{1}}} d x d \tau,
\end{aligned}
$$

and using the obvious inequality,

$$
\frac{1}{|x|^{a+a_{1}}(1+|x|)^{b_{1}}} \leq \frac{1}{\left|x_{k}\right|^{a_{1}}\left(1+\left|x_{k}\right|\right)^{b_{1}}\left|\bar{x}_{k}\right|^{a}},
$$

it follows that

$$
\begin{aligned}
\int_{\mathbb{R}^{n}} \mid & \left.\int_{\mathbb{R}^{n-1}} e^{i \bar{x}_{k} \cdot \bar{\eta}_{k}} F_{k}\left(x_{k}, \tau, \bar{\eta}_{k}\right) d \bar{\eta}_{k}\right|^{2} \frac{1}{|x|^{a+a_{1}}(1+|x|)^{b_{1}}} d x \\
= & \int_{\mathbb{R}^{n}}\left|\int_{\mathbb{R}^{n-1}} e^{i \bar{x}_{k} \cdot \bar{\eta}_{k}}\left(\sum_{l=1}^{\infty} F_{k, l}\left(x_{k}, \tau, \bar{\eta}_{k}\right)\right) d \bar{\eta}_{k}\right|^{2} \frac{1}{|x|^{a+a_{1}}(1+|x|)^{b_{1}}} d x \\
\leq & \int_{\mathbb{R}^{n}}\left|\int_{\mathbb{R}^{n-1}} e^{i \bar{x}_{k} \cdot \bar{\eta}_{k}}\left(\sum_{l=1}^{\infty} F_{k, l}\left(x_{k}, \tau, \bar{\eta}_{k}\right)\right) d \bar{\eta}_{k}\right|^{2} \\
& \times \frac{1}{\left|\bar{x}_{k}\right|^{a}\left|x_{k}\right|^{a_{1}}\left(1+\left|x_{k}\right|\right)^{b_{1}}} d \bar{x}_{k} d x_{k} \\
= & \int_{\mathbb{R}} \frac{1}{\left|x_{k}\right|^{a_{1}}\left(1+\left|x_{k}\right|\right)^{b_{1}}} \\
& \times\left(\int_{\mathbb{R}^{n-1}}\left|\int_{\mathbb{R}^{n-1}} e^{i \bar{x}_{k} \cdot \bar{\eta}_{k}}\left(\sum_{l=1}^{\infty} F_{k, l}\left(x_{k}, \tau, \bar{\eta}_{k}\right)\right) d \bar{\eta}_{k}\right|^{2} \frac{1}{\left|\bar{x}_{k}\right|^{a}} d \bar{x}_{k}\right) d x_{k} .
\end{aligned}
$$

By Lemma 2.1 with $b=0$ and $s_{0}=a / 2$, this is dominated by

$$
\begin{aligned}
& \int_{\mathbb{R}} \frac{1}{\left|x_{k}\right|^{a_{1}}\left(1+\left|x_{k}\right|\right)^{b_{1}}} \int_{\mathbb{R}^{n-1}}\left|\bar{\eta}_{k}\right|^{a}\left|\sum_{l=1}^{\infty} F_{k, l}\left(x_{k}, \tau, \bar{\eta}_{k}\right)\right|^{2} d \bar{\eta}_{k} d x_{k} \\
& \quad \leq C \int_{\mathbb{R}} \frac{1}{\left|x_{k}\right|^{a_{1}}\left(1+\left|x_{k}\right|\right)^{b_{1}}} \int_{\mathbb{R}^{n-1}} N \sum_{l=1}^{\infty}\left|F_{k, l}\left(x_{k}, \tau, \bar{\eta}_{k}\right)\right|^{2}\left|\bar{\eta}_{k}\right|^{a} d \bar{\eta}_{k} d x_{k},
\end{aligned}
$$


where the last inequality follows from the fact that

$$
\sum_{l=1}^{\infty} \chi_{B_{k, l}}\left(\bar{\eta}_{k}, \tau\right) \leq N, \quad \bar{\eta}_{k} \in \mathbb{R}^{n-1} \backslash E,
$$

which in turn follows from hypothesis (ii).

Since $0<a_{1}<1, a_{1}+b_{1}>1$

$$
\int_{\mathbb{R}} \frac{1}{\left|x_{k}\right|^{a_{1}}\left(1+\left|x_{k}\right|\right)^{b_{1}}} d x_{k}<+\infty,
$$

and hence the right side of (2.15) is less than or equal to

$$
C \int_{\mathbb{R}^{n-1}}\left(\sum_{l=1}^{\infty} \chi_{B_{k, l}}\left(\bar{\eta}_{k}, \tau\right)\left|G_{k, l}\left(\bar{\eta}_{k}, \tau\right)\right|^{2}\right) d \bar{\eta}_{k} .
$$

Substituting (2.15) and (2.16) into (2.14), we get

$$
\begin{aligned}
& \int_{\mathbb{R}^{n}} \int_{\mathbb{R}^{\prime}}\left(1+\tau^{2}\right)^{s_{0}}\left|\left(S_{\Omega, D} f\right)^{\uparrow}(x, \cdot)(\tau)\right|^{2} \frac{1}{|x|^{a+a_{1}}(1+|x|)^{b_{1}}} d x d t \\
& \leq C \int_{\mathbb{R}^{n}}\left(1+\tau^{2}\right)^{s_{0}} \sum_{k=1}^{n} \sum_{l=1}^{\infty} \chi_{B_{k, l}}\left(\bar{\eta}_{k}, \tau\right)\left|\bar{\eta}_{k}\right|^{a}\left|G_{k, l}\left(\bar{\eta}_{k}, \tau\right)\right|^{2} d \bar{\eta}_{k} d \tau \\
&=C \sum_{k=1}^{n} \sum_{l=1}^{\infty} \int_{\mathbb{R}^{n}}\left(1+\left|\eta_{k}\right|^{2}\right)^{s_{0}}\left|\bar{\eta}_{k}\right|^{a} \chi_{B_{k, l}}(\eta)\left|G_{k, l}\left(\bar{\eta}_{k}, \eta_{k}\right)\right|^{2} d \eta \\
&=C \sum_{k=1}^{n} \sum_{l=1}^{\infty} \int_{B_{k, l}}\left(1+\left|\eta_{k}\right|^{2}\right)^{s_{0}}\left|\bar{\eta}_{k}\right|^{a}\left|G_{k, l}\left(\bar{\eta}_{k}, \eta_{k}\right)\right|^{2} d \eta \\
&=C \sum_{k=1}^{n} \sum_{l=1}^{\infty} \int_{B_{k, l}}\left(1+\left|\eta_{k}\right|^{2}\right)^{s_{0}}\left|\bar{\eta}_{k}\right|^{a} \\
& \quad \times\left|\hat{f}\left(\left(T^{(k)}\right)^{-1}(\eta)\right)\right|^{2}\left|\frac{\partial \Omega}{\partial \xi_{k}}\left(\left(T^{(k)}\right)^{-1}(\eta)\right)\right|^{-2} d \eta \\
&= C \sum_{k=1}^{n} \sum_{l=1}^{\infty} \int_{A_{k, l}}\left(1+|\Omega(\xi)|^{2}\right)^{s_{0}}\left|\bar{\xi}_{k}\right|^{a}|\hat{f}(\xi)|^{2}\left|\frac{\partial \Omega}{\partial \xi_{k}}(\xi)\right|^{-1} d \xi \\
& \leq C \sum_{k=1}^{n} \sum_{l=1}^{\infty} \int_{A_{k, l}}\left(1+|\Omega(\xi)|^{2}\right)^{s_{0}}|\xi|^{a}|\hat{f}(\xi)|^{2}|\nabla \Omega(\xi)|^{-1} d \xi \\
&= C \int_{D}\left(1+|\Omega(\xi)|^{2}\right)^{s_{0}}|\xi|^{a}|\nabla \Omega(\xi)|^{-1}|\hat{f}(\xi)|^{2} d \xi
\end{aligned}
$$

This proves the first part of the lemma.

To prove (II), observe that the proof of Part (I) required assumption (ii) only in the proof of the estimate (2.15). But if $\Omega$ is a non-constant polynomial, the terms in the sum of the integrand of (2.15) do not exceed the degree of $\Omega$ and hence the inequality (2.15) is obviously satisfied. The remaining argument of the proof is then identical to that of (I).

Remark 2.1. If in Lemma 2.5, $D=\mathbb{R}^{n}, 0<\delta<1, a_{1}=1-\delta, b_{1}=2 \delta, 0 \leq a<n-1$, and $s_{0} \in \mathbb{R}$, then the inequality (2.11) can be reformulated as 


$$
\begin{aligned}
& \left(\int_{\mathbb{R}^{n}} \int_{\mathbb{R}}\left|\left(S_{\Omega} f\right)^{\curlyvee}(x, \cdot)(\tau)\right|^{2}\left(1+|\tau|^{2}\right)^{s_{0}} \frac{1}{|x|^{1+a}}\left(\frac{|x|}{1+|x|^{2}}\right)^{\delta} d \tau d x\right)^{1 / 2} \\
& \leq C\left(\int_{\mathbb{R}^{n}} \frac{\left(1+|\Omega(\xi)|^{2}\right)^{s_{0}}}{|\nabla \Omega(\xi)|}|\xi|^{a}|\hat{f}(\xi)|^{2} d \xi\right)^{1 / 2}
\end{aligned}
$$

If $\Omega$ is a radial function, then (2.7) of Lemma 2.4 shows that this estimate holds with $\delta=0$, however $a$ may not be zero in this case. Hence for $0<a<n-1$, the case where $\Omega$ is radial may be viewed as the limiting case of the non-radial case.

\section{Main Results}

In [28] Vega proved that if $d>0, b>1$ and $s>b / 2$, there exists a constant $C>0$ independent of $f$, such that the estimate

$$
\left(\int_{\mathbb{R}^{n}}\left|\left(S_{d}^{* *} f\right)(x)\right|^{2}(1+|x|)^{-b} d x\right)^{1 / 2} \leq C\|f\|_{H_{s}}
$$

holds for $f \in \mathcal{S}\left(\mathbb{R}^{n}\right)$. The main objective of this section is to extend the estimate (3.1) to the maximal operator $S_{\Omega}^{* *}$, where $\Omega$ is a general phase function. Specifically we shall establish estimates of the type

$$
\left(\int_{\mathbb{R}^{n}}\left|\left(S_{\Omega}^{* *} f\right)(x)\right|^{2}|x|^{-a}(1+|x|)^{-b} d x\right)^{1 / 2} \leq C\|f\|_{H_{s}}
$$

where $a \geq 0, b \geq 0, s \geq 0$ and $\Omega$ are continuously differentiable functions on $\mathbb{R}^{n} \backslash\{0\}$.

The first theorem in this direction considers the case where $\Omega$ is radial and has polynomial growth. Recall that a function $\varphi$ has polynomial growth if $|\varphi(t)| \leq$ $C(1+t)^{m}, t>0$, where $m>0$ is an integer.

Theorem 3.1. Let $n \geq 2, \Omega(\xi)=\varphi(|\xi|)$, where $\varphi \in C^{1}[0,+\infty)$ has polynomial growth. If $\left|\varphi^{\prime}(t)\right|>0$, and $0<a<n-1, \varepsilon>0$, then

$$
\begin{aligned}
& \left(\int_{\mathbb{R}^{n}}\left|\left(S_{\Omega}^{* *} f\right)(x)\right|^{2} \frac{d x}{|x|^{1+a}}\right)^{1 / 2} \\
& \quad \leq C\left(\int_{\mathbb{R}^{n}} \frac{\sqrt{1+|\varphi(|\xi|)|^{2}}}{\left|\varphi^{\prime}(|\xi|)\right|}|\xi|^{a}\left(1+|\xi|^{2}\right)^{\varepsilon}|\hat{f}(\xi)|^{2} d \xi\right)^{1 / 2}
\end{aligned}
$$

holds for $f \in \mathcal{S}\left(\mathbb{R}^{n}\right)$.

Proof. Fix $f \in \mathcal{S}\left(\mathbb{R}^{n}\right)$ and assume $|\varphi(|\xi|)| \leq C(1+|\xi|)^{m}, \xi \in \mathbb{R}^{n}$.

Let $\varepsilon_{1}=\varepsilon / m$, and $s_{0}=1 / 2+\varepsilon_{1}$, then by Lemma 2.4

$$
\begin{aligned}
\left(\int_{\mathbb{R}^{n}}\right. & \left.\left(\int_{\mathbb{R}}\left(1+\tau^{2}\right)^{s_{0}}\left|\left(S_{\Omega} f\right)^{\uparrow}(x, \cdot)(\tau)\right|^{2} d \tau\right) \frac{d x}{|x|^{1+a}}\right)^{1 / 2} \\
\leq & C\left(\int_{\mathbb{R}^{n}} \frac{\left(1+|\varphi(|\xi|)|^{2}\right)^{s_{0}}}{\left|\varphi^{\prime}(|\xi|)\right|}|\xi|^{a}|\hat{f}(\xi)|^{2} d \xi\right)^{1 / 2} \\
\quad= & C\left(\int_{\mathbb{R}^{n}} \frac{\sqrt{1+|\varphi(|\xi|)|^{2}}}{\left|\varphi^{\prime}(|\xi|)\right|}\left(1+|\varphi(|\xi|)|^{2}\right)^{\varepsilon_{1}}|\xi|^{a}|\hat{f}(\xi)|^{2} d \xi\right)^{1 / 2} .
\end{aligned}
$$


But since

$$
\begin{gathered}
\left(1+|\varphi(|\xi|)|^{2}\right)^{\varepsilon_{1}} \leq\left(1+C^{2}(1+|\xi|)^{2 m}\right)^{\varepsilon_{1}} \leq C\left(1+|\xi|^{2}\right)^{m \varepsilon_{1}} \leq C\left(1+|\xi|^{2}\right)^{\varepsilon}, \\
\left(\int_{\mathbb{R}^{n}}\left(\int_{\mathbb{R}^{2}}\left(1+\tau^{2}\right)^{s_{0}}\left|\left(S_{\Omega} f\right)^{\curlyvee}(x, \cdot)(\tau)\right|^{2} d \tau\right) \frac{d x}{|x|^{1+a}}\right)^{1 / 2} \\
\leq C\left(\int_{\mathbb{R}^{n}} \frac{\sqrt{1+|\varphi(|\xi|)|^{2}}}{\left|\varphi^{\prime}(|\xi|)\right|}\left(1+|\xi|^{2}\right)^{\varepsilon}|\xi|^{a}|\hat{f}(\xi)|^{2} d \xi\right)^{1 / 2}<+\infty .
\end{gathered}
$$

It follows from (3.4) and Hölder's inequality that $\left(S_{\Omega} f\right)^{\wedge}(x, \cdot) \in L^{1}(\mathbb{R})$. Hence $F(x, t) \equiv \frac{1}{2 \pi} \int_{\mathbb{R}} e^{i t \tau}\left(S_{\Omega} f\right)^{\curlyvee}(x, \cdot)(\tau) d \tau$ exists and since for any $g \in \mathcal{S}(\mathbb{R})$

$$
\int_{\mathbb{R}} F(x, t) \hat{g}(t) d t=\int_{\mathbb{R}}\left(S_{\Omega} f\right)^{\uparrow}(x, \cdot)(\tau) g(\tau) d \tau=\int_{\mathbb{R}}\left(S_{\Omega} f\right)(x, t) \hat{g}(t) d t,
$$

it follows that $F(x, t)=\left(S_{\Omega} f\right)(x, t)$. Therefore by Hölder's inequality

$$
\begin{aligned}
\left(S_{\Omega}^{* *} f\right)(x) & =\sup _{t \in \mathbb{R}}\left|\left(S_{\Omega} f\right)(x, t)\right| \leq \frac{1}{2 \pi} \int_{\mathbb{R}}\left|\left(S_{\Omega} f\right)^{\curlyvee}(x, \cdot)(\tau)\right| d \tau \\
& \leq \frac{1}{2 \pi}\left(\int_{\mathbb{R}}\left(1+\tau^{2}\right)^{-s_{0}} d \tau\right)^{1 / 2}\left(\int_{\mathbb{R}}\left(1+\tau^{2}\right)^{s_{0}}\left|\left(S_{\Omega} f\right)^{\curlyvee}(x, \cdot)(\tau)\right|^{2} d \tau\right)^{1 / 2}
\end{aligned}
$$

and since the first integral is finite, we obtain on integrating that the left side of (3.3) is dominated by (3.4). Hence the result follows.

If $\Omega$ is not necessarily radial, but still has polynomial growth one has

Theorem 3.2. Let $n \geq 2, D \subset \mathbb{R}^{n}$ open, and $\Omega \in C^{1}(D)$, where $\Omega$ has polynomial growth. If $\Omega$ satisfies either conditions (i) or (ii) of Lemma 2.5, or is a nonconstant polynomial satisfying (i) of Lemma 2.5, then given $0<a_{1}<1, b_{1}>0$, with $a_{1}+b_{1}>1$ and $0 \leq a<n-1, \varepsilon>0$, there exists a constant $C>0$ independent of $f \in \mathcal{S}\left(\mathbb{R}^{n}\right)$, such that

$$
\begin{aligned}
& \left(\int_{\mathbb{R}^{n}}\left|\left(S_{\Omega, D}^{* *} f\right)(x)\right|^{2} \frac{d x}{|x|^{a+a_{1}}(1+|x|)^{b_{1}}}\right)^{1 / 2} \\
& \quad \leq C\left(\int_{D} \frac{\sqrt{1+|\Omega(\xi)|^{2}}}{|\nabla \Omega(\xi)|}|\xi|^{a}\left(1+|\xi|^{2}\right)^{\varepsilon}|\hat{f}(\xi)|^{2} d \xi\right)^{1 / 2} .
\end{aligned}
$$

Proof. The proof is similar to that of Theorem 3.1 and is given for completeness only. Fix $f \in \mathcal{S}\left(\mathbb{R}^{n}\right)$ and assume the right side of (3.5) is finite. Since $\Omega$ has polynomial growth, let $|\Omega(\xi)| \leq C(1+|\xi|)^{m}, \xi \in D$, and $\varepsilon_{1}=\varepsilon / m, s_{0}=1 / 2+\varepsilon_{1}$. 
Then Lemma 2.5 yields

$$
\begin{aligned}
\left(\int_{\mathbb{R}^{n}}\right. & \left.\left(\int_{\mathbb{R}}\left(1+\tau^{2}\right)^{s_{0}}\left|\left(S_{\Omega, D} f\right)^{\wedge}(x, \cdot)(\tau)\right|^{2} d \tau\right) \frac{d x}{|x|^{a+a_{1}}(1+|x|)^{b_{1}}} d x\right)^{1 / 2} \\
& \leq C\left(\int_{D} \frac{\left(1+|\Omega(\xi)|^{2}\right)^{s_{0}}}{|\nabla \Omega(\xi)|}|\xi|^{a}|\hat{f}(\xi)|^{2} d \xi\right)^{1 / 2} \\
& =C\left(\int_{D} \frac{\sqrt{1+|\Omega(\xi)|^{2}}}{|\nabla \Omega(\xi)|}\left(1+|\Omega(\xi)|^{2}\right)^{\varepsilon_{1}}|\xi|^{a}|\hat{f}(\xi)|^{2} d \xi\right)^{1 / 2} \\
& \leq C\left(\int_{D} \frac{\sqrt{1+|\Omega(\xi)|^{2}}}{|\nabla \Omega(\xi)|}\left(1+|\xi|^{2}\right)^{\varepsilon}|\xi|^{a}|\hat{f}(\xi)|^{2} d \xi\right)^{1 / 2}<+\infty
\end{aligned}
$$

Fubini's theorem and (3.6) shows that for almost all $x \in \mathbb{R}^{n}$,

$$
\int_{\mathbb{R}}\left(1+\tau^{2}\right)^{s_{0}}\left|\left(S_{\Omega, D} f\right) \uparrow(x, \cdot)(\tau)\right|^{2} d \tau<+\infty .
$$

As in the proof of Theorem 3.1,

$$
\left|\left(S_{\Omega, D}^{* *} f\right)(x)\right|^{2} \leq C \int_{\mathbb{R}}\left|\left(S_{\Omega, D} f\right)^{\wedge}(x, \cdot)(\tau)\right|^{2}\left(1+\tau^{2}\right)^{s_{0}} d \tau,
$$

and integrating with respect to $\frac{1}{|x|^{a+a_{1}(1+|x|)^{b_{1}}}}$ and applying (3.6), the result follows.

It is not difficult to see from this proof that the integral on the right of (3.5) can be replaced by

$$
\left(\int_{D}\left(1+|\Omega(\xi)|^{2}\right)^{s_{0}}|\nabla \Omega(\xi)|^{-1}|\xi|^{a}|\hat{f}(\xi)|^{2} d \xi\right)^{1 / 2}
$$

where $s_{0}>1 / 2$.

Before proving the first main result we need a lemma.

Lemma 3.1. Let $n \geq 2$, and $D$ a measurable set in $\mathbb{R}^{n}$ with finite Lebesgue measure. Let $\Omega$ be any real-valued measurable function defined on $D$. Then for $0<a<n, b>0$, with $a+b>n$, and $s>0$, there exists a constant $C>0$ independent of $f \in \mathcal{S}\left(\mathbb{R}^{n}\right)$, such that

$$
\left(\int_{\mathbb{R}^{n}}\left|\left(S_{\Omega, D}^{* *} f\right)(x)\right|^{2} \frac{d x}{|x|^{a}(1+|x|)^{b}}\right)^{1 / 2} \leq C\|f\|_{H_{s}} .
$$

Proof. Since

$$
\begin{aligned}
\left|\left(S_{\Omega, D}^{* *} f\right)(x)\right| & \leq(2 \pi)^{-n} \int_{D}|\hat{f}(\xi)| d \xi \\
& \leq(2 \pi)^{-n}\left(\int_{D}\left(1+|\xi|^{2}\right)^{-s} d \xi\right)^{1 / 2}\left(\int_{D}\left(1+|\xi|^{2}\right)^{s}|\hat{f}(\xi)|^{2} d \xi\right)^{1 / 2} \\
& \leq(2 \pi)^{-n}\left(\int_{D} d \xi\right)^{1 / 2}\left(\int_{\mathbb{R}^{n}}\left(1+|\xi|^{2}\right)^{s}|\hat{f}(\xi)|^{2} d \xi\right)^{1 / 2} \leq C\|f\|_{H_{s}} .
\end{aligned}
$$

But since

$$
\left(\int_{\mathbb{R}^{n}} \frac{1}{|x|^{a}(1+|x|)^{b}} d x\right)^{1 / 2}<+\infty
$$

(3.7) follows. 
In the next theorem sharp estimates are proved for the global maximal function whose phase function is a polynomial of principal type.

Theorem 3.3. Let $n \geq 2, \xi \in \mathbb{R}^{n}$ and $\Omega(\xi)=\sum_{k=0}^{N} P_{k}(\xi)$, where $P_{k}, \quad k=$ $0,1,2, \ldots, N$, are real homogeneous polynomials of order $k$. If $\Omega$ is of principal type, that is, $\left|\nabla P_{N}(\xi)\right|^{2}$ is elliptic, then for $1<a<n, b>0$, with $a+b>n$, and $f \in \mathcal{S}\left(\mathbb{R}^{n}\right)$, (3.2) is satisfied for $s>a / 2$ and fails for $s<a / 2$.

Proof. Since $P_{k}$ are homogeneous of order $k$, it follows that $\left|\nabla P_{k}\right|$ are homogeneous functions of order $k-1$. Hence there exist constants $C_{i}>0, i=1,2$, such that for $1 \leq k \leq N-1$ and $\xi \in \mathbb{R}^{n},\left|\nabla P_{k}(\xi)\right| \leq C_{1}|\xi|^{k-1}$, and since $\left|\nabla P_{N}\right|^{2}$ is elliptic $\left|\nabla P_{N}(\xi)\right| \geq C_{2}|\xi|^{N-1}$. If $|\xi|>R, R>0$, sufficiently large, then

$$
|\nabla \Omega(\xi)| \geq C|\xi|^{N-1}-C \sum_{k=1}^{N-1}|\xi|^{k-1} \geq C|\xi|^{N-1}>1
$$

and

$$
|\Omega(\xi)| \leq \sum_{k=1}^{N}\left|P_{k}(\xi)\right| \leq C \sum_{k=1}^{N}|\xi|^{k} \leq C|\xi|^{N}
$$

Combining these estimates yields for $|\xi|>R$

$$
\frac{|\Omega(\xi)|}{|\nabla \Omega(\xi)|} \leq C|\xi|
$$

Let $B(R)=\{\xi ;|\xi| \leq R\}$, and $B^{c}(R)$ its complement, then clearly

$$
\left(S_{\Omega}^{* *} f\right)(x) \leq\left(S_{\Omega, B(R)}^{* *} f\right)(x)+\left(S_{\Omega, B^{c}(R)}^{* *} f\right)(x) .
$$

By Lemma 3.1

$$
\left(\int_{\mathbb{R}^{n}}\left|\left(S_{\Omega, B(R)}^{* *} f\right)(x)\right|^{2} \frac{1}{|x|^{a}(1+|x|)^{b}} d x\right)^{1 / 2} \leq C\|f\|_{H_{s}}
$$

so it remains to show that for $s>a / 2,0<a<n$, and $b>0$ with $a+b>n$,

$$
\left(\int_{\mathbb{R}^{n}}\left|\left(S_{\Omega, B^{c}(R)}^{* *} f\right)(x)\right|^{2} \frac{1}{|x|^{a}(1+|x|)^{b}} d x\right)^{1 / 2} \leq C\|f\|_{H_{s}},
$$

for then the positive result follows from Minkowski's inequality. From Theorem 3.2, it follows that for $0 \leq a<n-1,0 \leq a_{1}<1, b_{1}>0$ with $a_{1}+b_{1}>1$ and $\varepsilon>0$,

$$
\begin{aligned}
\int_{\mathbb{R}^{n}} & \left|\left(S_{\Omega, B^{c}(R)}^{* *} f\right)(x)\right|^{2} \frac{1}{|x|^{a+a_{1}}(1+|x|)^{b_{1}}} d x \\
& \leq C \int_{B^{c}(R)} \frac{\sqrt{1+|\Omega(\xi)|^{2}}}{|\nabla \Omega(\xi)|}\left(1+|\xi|^{2}\right)^{\varepsilon}\left(1+|\xi|^{2}\right)^{a / 2}|\hat{f}(\xi)|^{2} d \xi \\
& \leq C \int_{B^{c}(R)}\left[\sqrt{\frac{1}{|\nabla \Omega(\xi)|^{2}}+\frac{|\Omega(\xi)|^{2}}{|\nabla \Omega(\xi)|^{2}}}\right]\left(1+|\xi|^{2}\right)^{\varepsilon+a / 2}|\hat{f}(\xi)|^{2} d \xi
\end{aligned}
$$


Applying (3.8), (3.9), the last integral is dominated by

$$
\begin{aligned}
\int_{B^{c}(R)} & {\left[\sqrt{1+C^{2}|\xi|^{2}}\right]\left(1+|\xi|^{2}\right)^{a / 2+\varepsilon}|\hat{f}(\xi)|^{2} d \xi } \\
\leq & C \int_{B^{c}(R)}\left(1+|\xi|^{2}\right)^{1 / 2+a / 2+\varepsilon}|\hat{f}(\xi)|^{2} d \xi \\
\leq & C\|f\|_{H_{1 / 2+a / 2+\varepsilon}}^{2}=C\|f\|_{H_{\left(a+a_{1}\right) / 2+\left(1-a_{1}\right) / 2+\varepsilon}}^{2}
\end{aligned}
$$

Choosing $a_{1}$ and $b_{1}$ so that $\left(1-a_{1}\right) / 2+\varepsilon$ is small, (3.10) follows with $a+a_{1}$ replaced by $a$.

To show that (3.2) fails if $s<a / 2$, observe first that

$$
\begin{aligned}
\left(S_{\Omega}^{* *} f\right)(x) & =(2 \pi)^{-n} \sup _{t \in \mathbb{R}}\left|\int_{\mathbb{R}^{n}} e^{i x \cdot \xi} e^{i t \Omega(\xi)} \hat{f}(\xi) d \xi\right| \\
& \geq(2 \pi)^{-n}\left|\int_{\mathbb{R}^{n}} e^{i x \cdot \xi} \hat{f}(\xi) d \xi\right|=|f(x)|,
\end{aligned}
$$

where the last equality is the Fourier inversion theorem.

Suppose (3.2) holds for $f \in \mathcal{S}\left(\mathbb{R}^{n}\right)$, then substituting the last estimate into (3.2), we obtain

$$
\left(\int_{\mathbb{R}^{n}}|f(x)|^{2} \frac{d x}{|x|^{a}(1+|x|)^{b}}\right)^{1 / 2} \leq C\left(\int_{\mathbb{R}^{n}}\left(1+|\xi|^{2}\right)^{s}|\hat{f}(\xi)|^{2} d \xi\right)^{1 / 2}
$$

which is equivalent (via the inverse Fourier transform) to

$$
\left(\int_{\mathbb{R}^{n}}|\hat{f}(x)|^{2} \frac{d x}{|x|^{a}(1+|x|)^{b}}\right)^{1 / 2} \leq C\left(\int_{\mathbb{R}^{n}}\left(1+|x|^{2}\right)^{s}|f(x)|^{2} d x\right)^{1 / 2} .
$$

But by Lemma 2.1 , with $p=2$, this inequality holds if and only if $s \geq a / 2$, i.e. (3.2) fails if $s<a / 2$.

This proves the theorem.

If $\Omega$ is a real homogeneous polynomial of principal type, then $|\nabla \Omega|^{2}$ is an elliptic homogeneous polynomial and hence has no zeros on the unit sphere $S^{n-1}$ in $\mathbb{R}^{n}$. If $\Omega$ is a real homogeneous polynomial but not of principal type, then $|\nabla \Omega|$ has zeros on the unit sphere $S^{n-1}$. In this case sharp estimates of the form (3.2) can still be proved provided the zeros of $|\nabla \Omega|$ on $S^{n-1}$ are of regular type.

Let $g$ be a continuous function on $S^{n-1}$, such that $g\left(\xi_{0}^{\prime}\right)=0$. If $\theta$ is the angle between $\xi_{0}^{\prime}$ and $\xi^{\prime}, \xi^{\prime} \in S^{n-1}$, then $\xi_{0}^{\prime}$ is called a regular zero of order $\beta$, if

$$
\lim _{\theta \rightarrow 0} \frac{\left|g\left(\xi^{\prime}\right)-g\left(\xi_{0}^{\prime}\right)\right|}{\theta^{\beta}}=\lim _{\theta \rightarrow 0} \frac{\left|g\left(\xi^{\prime}\right)\right|}{\theta^{\beta}}=0 .
$$

Theorem 3.4. Let $n \geq 2$, and $\Omega$ a real homogeneous polynomial of order $m$ such that $|\nabla \Omega(\xi)|$ has only finitely many zeros, $\xi_{1}^{\prime}, \xi_{2}^{\prime}, \ldots, \xi_{l}^{\prime}$, on the unit sphere $S^{n-1}$ in $\mathbb{R}^{n}$, which are of regular type of order $\alpha_{j} \leq\left(1-\frac{1}{n}\right) m, j=1,2, \ldots, l$. Then for $1<a<n, b>0$, with $a+b>n$, the estimate (3.2) with $f \in \mathcal{S}\left(\mathbb{R}^{n}\right)$ holds for $s>a / 2$ and fails for $s<a / 2$.

Proof. We may assume that $|\nabla \Omega|$ has only one zero, since a repetition of the proof yields the general case.

Without loss of generality, assume also that the only zero of $|\nabla \Omega|$ on the unit sphere $S^{n-1}$ is $\xi_{N}^{\prime}=(0,0, \ldots, 0,1)$ and that $\xi_{N}^{\prime}$ is of regular type with order $m^{*} \leq(1-1 / n) m$. 
Let $k \geq 1$ be fixed and define $\theta_{k}$ and $A_{k}^{\prime}$ by

$$
\theta_{k}=k^{-n /(n-1)}(\ln (k+2))^{-3}, \quad A_{k}^{\prime}=\left\{\xi^{\prime} ; \xi^{\prime} \in S^{n-1}, 0 \leq \theta \leq \theta_{k}\right\},
$$

where $\theta$ is the angle between $\xi_{N}^{\prime}$ and $\xi^{\prime}$, that is, $\xi_{N}^{\prime} \cdot \xi^{\prime}=\cos \theta$. Clearly

$$
A_{1}^{\prime} \supset A_{2}^{\prime} \supset A_{3}^{\prime} \supset \cdots \text {. }
$$

Let

$$
\begin{cases}B_{0}=\{\xi ;|\xi| \leq 1\}, & \\ B_{k}=\left\{\xi ; \xi=|\xi| \xi^{\prime}, k<|\xi| \leq k+1, \xi^{\prime} \in A_{k}^{\prime}\right\}, & (k \geq 1), \\ D_{k}\left\{\xi ; \xi=|\xi| \xi^{\prime}, k<|\xi| \leq k+1, \xi^{\prime} \in A_{1}^{\prime} \backslash A_{k}^{\prime}\right\}, & (k \geq 2),\end{cases}
$$

and define

$$
\left\{\begin{array}{l}
B=\bigcup_{k \geq 0} B_{k}, \\
E=\left\{\bar{\xi} ; \xi=|\xi| \xi^{\prime},|\xi|>1, \xi^{\prime} \in S^{n-1} \backslash A_{1}^{\prime},\right\},
\end{array}\right.
$$

then $\mathbb{R}^{n}=B \cup E \cup \bigcup_{k \geq 2} D_{k}$, and therefore

$$
\begin{aligned}
\left(S_{\Omega}^{* *} f\right)(x) & =\sup _{t \in R}\left|\left(S_{\Omega} f\right)(x, t)\right| \\
& \leq\left(S_{\Omega, B}^{* *} f\right)(x)+\left(S_{\Omega, E}^{* *} f\right)(x)+\left(S_{\Omega, \bigcup_{k \geq 2} D_{k}}^{* *} f\right)(x) .
\end{aligned}
$$

We shall estimate $S_{\Omega, B}^{* *}, S_{\Omega, E}^{* *}, S_{\Omega, \bigcup_{k}>2}^{* *} D_{k}$ separately.

First, we show that $B$ has finite Lebesgue measure. To prove this, introduce polar coordinates on $S^{n-1}$ as follows:

$$
\begin{aligned}
& \xi_{n}=\cos \theta_{1}, \\
& \xi_{n-1}=\sin \theta_{1} \cos \theta_{2}, \\
& \cdots, \\
& \xi_{n-k+1}=\sin \theta_{1} \sin \theta_{2} \cdots \sin \theta_{k-1} \cos \theta_{k}, \\
& \cdots, \\
& \xi_{2}=\sin \theta_{1} \cdots \sin \theta_{n-2} \cos \theta_{n-1}, \\
& \xi_{1}=\sin \theta_{1} \cdots \sin \theta_{n-2} \sin \theta_{n-1} .
\end{aligned}
$$

Let the surface measure on $S^{n-1}$ be denoted by $d \sigma_{n-1}$, then it follows from (3.11) that

$$
\begin{aligned}
\left|B_{k}\right| & =\int_{k}^{k+1} r^{n-1} d r \int_{A_{k}^{\prime}} d \sigma_{n-1}\left(\theta_{1}, \ldots, \theta_{n-1}\right) \\
& =\int_{k}^{k+1} r^{n-1} \int_{0}^{\theta_{k}}\left(\sin \theta_{1}\right)^{n-2} d \theta_{1} \int_{S^{n-2}} d \sigma_{n-2}\left(\theta_{2}, \ldots, \theta_{n-1}\right) d r \\
& \leq C k^{n-1} \int_{0}^{\theta_{k}} \theta_{1}^{n-2} d \theta_{1}\left|S^{n-2}\right| \leq C k^{n-1} \theta_{k}^{n-1}\left|S^{n-2}\right| \\
& \leq C k^{n-1}\left(k^{-n /(n-1)}(\ln (k+2))^{-3}\right)^{n-1} \leq C \frac{1}{k(\ln (k+2))^{3(n-1)}},
\end{aligned}
$$

where $\left|S^{n-2}\right|$ is the surface area of $S^{n-2}$ and $C>0$ is independent of $k$.

Therefore

$$
|B|=\left|B_{0}\right|+\sum_{k \geq 1}\left|B_{k}\right| \leq C\left(1+\sum_{k=1}^{\infty} \frac{1}{k(\ln (k+2))^{3(n-1)}}\right)<\infty .
$$


Now Lemma 3.1 applies and for any $1<a<n, b>0, s>0$ with $a+b>n$

$$
\left(\int_{\mathbb{R}^{n}}\left|S_{\Omega, B}^{* *} f(x)\right|^{2} \frac{1}{|x|^{a}(1+|x|)^{b}} d x\right)^{1 / 2} \leq C\|f\|_{H_{s}}
$$

Next we estimate $S_{\Omega, \bigcup_{k \geq 2} D_{k}}^{* *}$. Since $\xi_{N}^{\prime}$ is a regular zero of $|\nabla \Omega|$ of order $m^{*}$, there is a constant $C>0$ independent of $k$, such that $\left|\nabla \Omega\left(\xi^{\prime}\right)\right| \geq C \theta^{m^{*}}$, where $\theta$ is the angle between $\xi_{N}^{\prime}$ and $\xi^{\prime}$. Hence for $\xi \in D_{k}$, (cf. (3.12), (3.11))

$$
\begin{aligned}
|\nabla \Omega(\xi)| & =|\xi|^{m-1}\left|\nabla \Omega\left(\xi^{\prime}\right)\right| \geq C|\xi|^{m-1} \theta^{m^{*}} \geq C k^{m-1} \theta_{k}^{m^{*}} \\
& \geq C k^{m-1}\left\{k^{-n /(n-1)}(\ln (k+2))^{-3}\right\}^{m^{*}} \\
& \geq C k^{m-1-m^{*} n /(n-1)}\{\ln (k+2)\}^{-3 m^{*}},
\end{aligned}
$$

where $C>0$ is independent of $k$. Since $\Omega$ is homogeneous, Euler's equation $\nabla \Omega(\xi)$. $\xi=n \Omega(\xi)$ holds, and hence $n|\Omega(\xi)|=|\nabla \Omega(\xi) \cdot \xi| \leq|\xi||\nabla \Omega(\xi)|$, that is,

$$
\frac{|\Omega(\xi)|}{|\nabla \Omega(\xi)|} \leq(1 / n)|\xi|
$$

From this, (3.16) and the assumption $m^{*} \leq(1-1 / n) m$, one obtains for $\xi \in D_{k}$,

$$
\begin{aligned}
& \sqrt{\frac{1}{|\nabla \Omega(\xi)|^{2}}+\frac{|\Omega(\xi)|^{2}}{|\nabla \Omega(\xi)|^{2}}} \leq C \sqrt{k^{2+2 m^{*} n /(n-1)-2 m}[\ln (k+2)]^{6 m^{*}}+|\xi|^{2}} \\
& \quad \leq C \sqrt{|\xi|^{2}[\ln (|\xi|+2)]^{6 m^{*}}+|\xi|^{2}} \leq C\left(1+|\xi|^{2}\right)^{1 / 2}\left[1+(\ln (2+|\xi|))^{6 m^{*}}\right]^{1 / 2} .
\end{aligned}
$$

Applying Theorem 3.2 with $D=\bigcup_{k \geq 2} D_{k}$, it follows that for $0<a_{1}<1, b_{1}>0$ with $a_{1}+b_{1}>1$ and $0 \leq a<n-1, \varepsilon>0$,

$$
\begin{aligned}
\int_{\mathbb{R}^{n}} & \left|\left(S_{\Omega, \cup_{k \geq 2} D_{k}}^{* *} f\right)(x)\right|^{2} \frac{1}{|x|^{a+a_{1}}(1+|x|)^{b_{1}}} d x \\
& \leq C \int_{\bigcup_{k \geq 2} D_{k}} \frac{\sqrt{1+|\Omega(\xi)|^{2}}}{|\nabla \Omega(\xi)|}\left(1+|\xi|^{2}\right)^{a / 2+\varepsilon}|\hat{f}(\xi)|^{2} d \xi \\
& =C \sum_{k \geq 2} \int_{D_{k}} \frac{\sqrt{1+|\Omega(\xi)|^{2}}}{|\nabla \Omega(\xi)|}\left(1+|\xi|^{2}\right)^{a / 2+\varepsilon}|\hat{f}(\xi)|^{2} d \xi \\
& \leq C \sum_{k \geq 2} \int_{D_{k}}\left(1+|\xi|^{2}\right)^{a / 2+1 / 2+\varepsilon}\left[1+(\ln (2+|\xi|))^{6 m^{*}}\right]^{1 / 2}|\hat{f}(\xi)|^{2} d \xi \\
& =C \int_{\bigcup_{k \geq 2} D_{k}}\left(1+|\xi|^{2}\right)^{(a+1) / 2+\varepsilon}\left[1+\left(\ln (2+|\xi|)^{6 m^{*}}\right)\right]^{1 / 2}|\hat{f}(\xi)|^{2} d \xi \\
& \leq C \int_{\mathbb{R}^{n}}\left(1+|\xi|^{2}\right)^{(a+1) / 2+\varepsilon}\left[1+(\ln (2+|\xi|))^{6 m^{*}}\right]^{1 / 2}|\hat{f}(\xi)|^{2} d \xi \\
& \leq C \int_{\mathbb{R}^{n}}\left(1+|\xi|^{2}\right)^{(a+1) / 2+\varepsilon+\delta}|\hat{f}(\xi)|^{2} d \xi \leq C\|f\|_{H_{(a+1) / 2+\varepsilon+\delta}}^{2},
\end{aligned}
$$

where $\delta>0$. Arguing as in the proof of Theorem 3.3, there exists a constant $C>0$ independent of $f$, such that for $1<a<n, b>0$, with $a+b>n$ and $s>a / 2$

$$
\left(\int_{\mathbb{R}^{n}}\left|\left(S_{\Omega, \cup_{k \geq 2}^{*} D_{k}} f\right)(x)\right|^{2} \frac{1}{|x|^{a}(1+|x|)^{b}} d x\right)^{1 / 2} \leq C\|f\|_{H_{s}} .
$$


It remains to estimate $S_{\Omega, E}^{* *}$. If $\xi \in E$, then

$$
\left|\nabla \Omega\left(\xi^{\prime}\right)\right|=\inf _{\xi^{\prime} \in S^{n-1} \backslash A_{1}^{\prime}}\left|\nabla \Omega\left(\xi^{\prime}\right)\right| \geq C>0,
$$

and again by homogeneity of $\Omega$,

$$
\begin{aligned}
& \frac{\sqrt{1+|\Omega(\xi)|^{2}}}{|\nabla \Omega(\xi)|}=\sqrt{\frac{1}{|\nabla \Omega(\xi)|^{2}}+\frac{|\Omega(\xi)|^{2}}{|\nabla \Omega(\xi)|^{2}}} \\
& \quad \leq C \sqrt{\frac{1}{\left(|\xi|^{m-1}\left|\nabla \Omega\left(\xi^{\prime}\right)\right|\right)^{2}}+C|\xi|^{2}} \leq \sqrt{C|\xi|^{-2(m-1)}+C|\xi|^{2}} \\
& \quad \leq C \sqrt{1+|\xi|^{2}} .
\end{aligned}
$$

By Theorem 3.2, for $\varepsilon>0,0<a_{1}<1, b_{1}>0$, with $a_{1}+b_{1}>1$, and $0 \leq a<n-1$, there exists a constant $C>0$ independent of $f \in \mathcal{S}\left(\mathbb{R}^{n}\right)$, such that

$$
\begin{aligned}
& \left(\int_{\mathbb{R}^{n}}\left|\left(S_{\Omega, E}^{* *} f\right)(x)\right|^{2} \frac{1}{|x|^{a+a_{1}(1+|x|)^{b_{1}}}} d x\right)^{1 / 2} \\
& \quad \leq C\left(\int_{E} \frac{\sqrt{1+|\Omega(\xi)|^{2}}}{|\nabla \Omega(\xi)|}\left(1+|\xi|^{2}\right)^{\varepsilon}|\xi|^{a}|\hat{f}(\xi)|^{2} d \xi\right)^{1 / 2} \\
& \quad \leq C\left(\int_{E}\left(1+|\xi|^{2}\right)^{1 / 2+a / 2+\varepsilon}|\hat{f}(\xi)|^{2} d \xi\right)^{1 / 2} \leq C\|f\|_{H_{(1+a) / 2+\varepsilon}}
\end{aligned}
$$

Arguing again as in the proof of Theorem 3.3, it follows that for $1<a<n, b>0$, with $a+b>n$ and $s>a / 2$, there exists a constant $C>0$ independent of $f \in \mathcal{S}\left(\mathbb{R}^{n}\right)$, such that

$$
\left(\int_{\mathbb{R}^{n}}\left|\left(S_{\Omega, E}^{* *} f\right)(x)\right|^{2} \frac{d x}{|x|^{a}(1+|x|)^{b}}\right)^{1 / 2} \leq C\|f\|_{H_{s}} .
$$

Minkowski's inequality and (3.18), (3.17), (3.15) imply the result for $s>a / 2$.

The proof that (3.2) fails for $s<a / 2$, is identical to that of Theorem 3.3 and hence omitted.

Proposition 3.1. Let $n \geq 2$ and $\Omega(\xi)=\varphi(|\xi|)$ where $\varphi(t)=C t^{m}+S(t), m>$ $1, C>0$ and $S$ is a real-valued function. If

(i) $S \in C^{1}\left(t_{0}, \infty\right)$, for some $t_{0}>0$,

(ii) $\sup _{t>t_{0}}\left|S(t) / t^{m-1}\right|<\infty$,

(iii) $\sup _{t>t_{0}}\left|S^{\prime}(t) / t^{m-2}\right|<\infty$,

and $1<a<n, b>0$, with $a+b>n$, then (3.2) with $f \in \mathcal{S}\left(\mathbb{R}^{n}\right)$ holds if $s>a / 2$ and fails for $s<a / 2$.

Proof. The proof is similar to that of Theorem 3.3.

If $B=\left\{\xi ;|\xi| \leq t_{0}\right\}$ and $B^{c}$ its complement, then by Lemma 3.1 , for $0<a<$ $n, b>0$, with $a+b>n$ and $s>0$,

$$
\left(\int_{\mathbb{R}^{n}}\left|\left(S_{\Omega, B}^{* *} f\right)(x)\right|^{2} \frac{d x}{|x|^{a}(1+|x|)^{b}}\right)^{1 / 2} \leq C\|f\|_{H_{s}} .
$$

If for $1<a<n, b>0$, with $a+b>n$ and $s>a / 2$,

$$
\left(\int_{\mathbb{R}^{n}}\left|\left(S_{\Omega, B^{c}}^{* *} f\right)(x)\right|^{2} \frac{d x}{|x|^{a}(1+|x|)^{b}}\right)^{1 / 2} \leq C\|f\|_{H_{s}}
$$


holds, the result for $s>a / 2$ follows via Minkowski's inequality.

The properties of $\varphi$ imply that

$$
\begin{aligned}
\frac{\sqrt{1+|\Omega(\xi)|^{2}}}{|\nabla \Omega(\xi)|} & =\sqrt{\frac{1}{\left|\varphi^{\prime}(|\xi|)\right|^{2}}+\frac{|\varphi(|\xi|)|^{2}}{\left|\varphi^{\prime}(|\xi|)\right|^{2}}} \\
& =\sqrt{\frac{1}{\left[C m|\xi|^{m-1}+S^{\prime}(|\xi|)\right]^{2}}+\frac{\left[C|\xi|^{m}+S(|\xi|)\right]^{2}}{\left[C m|\xi|^{m-1}+S^{\prime}(|\xi|)\right]^{2}}} \\
& \leq C \sqrt{|\xi|^{-2(m-1)}+|\xi|^{2}} \leq C\left(1+|\xi|^{2}\right)^{1 / 2},
\end{aligned}
$$

and hence by Theorem 3.2, for $0<a_{1}<1, b_{1}>0$, with $a_{1}+b_{1}>1,0 \leq a<n-1$ and $\varepsilon>0$,

$$
\begin{aligned}
& \left(\int_{\mathbb{R}^{n}}\left|\left(S_{\Omega, B^{c}}^{* *} f\right)(x)\right|^{2} \frac{d x}{|x|^{a+a_{1}}(1+|x|)^{b_{1}}}\right)^{1 / 2} \\
& \quad \leq C\left(\int_{B^{c}} \sqrt{\frac{1}{\left|\varphi^{\prime}(|\xi|)\right|^{2}}+\frac{|\varphi(|\xi|)|^{2}}{\left|\varphi^{\prime}(|\xi|)\right|^{2}}}\left(1+|\xi|^{2}\right)^{\varepsilon}|\xi|^{a}|\hat{f}(\xi)|^{2} d \xi\right)^{1 / 2} \\
& \quad \leq C\left(\int_{B^{c}}\left(1+|\xi|^{2}\right)^{\varepsilon+1 / 2+a / 2}|\hat{f}(\xi)|^{2} d \xi\right)^{1 / 2} \\
& \quad \leq C\|f\|_{H_{\varepsilon+(1+a) / 2}} .
\end{aligned}
$$

Arguing as in the proof of Theorem 3.3, we obtain (3.19).

If $s<a / 2$, the result fails as was shown in the proof of Theorem 3.3.

Proposition 3.2. Let $n \geq 2$, and suppose $\Omega$ satisfies the conditions of any one of the Theorems 3.3, 3.4, or Proposition 3.1. Then for $p>2$, and $f \in \mathcal{S}\left(\mathbb{R}^{n}\right)$,

$$
\left(\int_{\mathbb{R}^{n}}\left|\left(S_{\Omega}^{* *} f\right)(x)\right|^{p} \frac{d x}{|x|^{a}(1+|x|)^{b}}\right)^{1 / p} \leq C\|f\|_{H_{s}}
$$

holds for $s>n(1 / 2-1 / p)+a / p$ and fails for $s<n(1 / 2-1 / p)+a / p$.

Proof. For $1<a<n, b>0$ with $a+b>n$ and $s_{1}=a / 2+\varepsilon, \varepsilon>0$,

$$
\left\|\left(S_{\Omega}^{* *} f\right)\right\|_{2, w} \leq C\|f\|_{H_{s_{1}}}
$$

holds for $f \in \mathcal{S}\left(\mathbb{R}^{n}\right)$, where $w(x)=|x|^{-a}(1+|x|)^{-b}$. Replacing $f$ by the inverse Fourier transform $\mathcal{F}^{-1} f$ in this estimate yields $\left\|\left(S_{\Omega}^{* *} \mathcal{F}^{-1} f\right)\right\|_{2, w} \leq C\|f\|_{2, v_{1}}$, where $v_{1}(x)=(1+|x|)^{s_{1}}$. On the other hand, for $s_{2}=n / 2+\varepsilon$, it follows from Schwartz's inequality that

$$
\begin{aligned}
\sup _{x \in \mathbb{R}^{n}} & \left|\left(S_{\Omega}^{* *} f\right)(x)\right| \leq(2 \pi)^{-n} \int_{\mathbb{R}^{n}}|\hat{f}(\xi)| d \xi \\
& \leq(2 \pi)^{-n}\left(\int_{\mathbb{R}^{n}}\left(1+|\xi|^{2}\right)^{-s_{2}} d \xi\right)^{1 / 2}\left(\int_{\mathbb{R}^{n}}\left(1+|\xi|^{2}\right)^{s_{2}}|\hat{f}(\xi)|^{2} d \xi\right)^{1 / 2} \\
& \leq C\|f\|_{H_{s_{2}}},
\end{aligned}
$$

and with $f$ replaced by $\mathcal{F}^{-1} f$ this is equivalent to $\left\|\left(S_{\Omega}^{* *} \mathcal{F}^{-1} f\right)\right\|_{\infty, w} \leq C\|f\|_{2, v_{2}}$, where $v_{2}(x)=(1+|x|)^{s_{2}}$. Applying the interpolation theorem with change of 
weights $([26])$, one obtains $\left\|\left(S_{\Omega}^{* *} \mathcal{F}^{-1} f\right)\right\|_{p, w} \leq C\|f\|_{2, v}$, where $1 / p=\theta / 2, v(x)=$ $v_{1}(x)^{\theta} v_{2}(x)^{(1-\theta)}, 0<\theta<1$. But this is equivalent to

$$
\left(\int_{\mathbb{R}^{n}}\left|\left(S_{\Omega}^{* *} f\right)(x)\right|^{p} \frac{d x}{|x|^{a}(1+|x|)^{b}}\right)^{1 / p} \leq C\|f\|_{H_{s}}
$$

with $s=(a / 2+\varepsilon)(2 / p)+(n / 2+\varepsilon)(1-2 / p)=n(1 / 2-1 / p)+a / p+\varepsilon$. Hence the first part is proved.

Conversely if (3.20) is assumed to hold for $f \in \mathcal{S}\left(\mathbb{R}^{n}\right)$, then as in the proof of Theorem 3.3,

$$
\left(\int_{\mathbb{R}^{n}}|f(x)|^{p} \frac{d x}{|x|^{a}(1+|x|)^{b}}\right)^{1 / p} \leq C\left(\int_{\mathbb{R}^{n}}\left(1+|x|^{2}\right)^{s}|\hat{f}(x)|^{2} d x\right)^{1 / 2} .
$$

But this inequality holds if and only if $s \geq n(1 / 2-1 / p)+a / p$ by Lemma 2.1.

This completes the proof of the proposition.

\section{Examples of Phase FUnCtions And EStimaes for $S_{d}^{* *}$}

First, examples of phase functions are given for which the hypotheses of Theorems 3.3 or 3.4 are satisfied. The following proposition comprises four examples.

Proposition 4.1. 1. Let $n=2$, and $\Omega\left(\xi_{1}, \xi_{2}\right)$ a non-constant real homogeneous harmonic polynomial on $\mathbb{R}^{2}$, then $|\nabla \Omega(\xi)|^{2}$ is an elliptic polynomial and therefore the conclusions of Theorem 3.3 and Theorem 3.4 hold for this phase function.

2. Let

$$
\begin{aligned}
\Omega\left(\xi_{1}, \xi_{2}\right) & =\frac{\left(\xi_{1}^{4 m}-\xi_{2}^{4 m}\right)}{\left(\xi_{1}^{2}+\xi_{2}^{2}\right)} \\
& =\left(\xi_{1}^{2}-\xi_{2}^{2}\right)\left\{\left(\xi_{1}^{m-1}\right)^{4}+\left(\xi_{1}^{m-2} \xi_{2}\right)^{4}+\cdots+\left(\xi_{1} \xi_{2}^{m-2}\right)^{4}+\left(\xi_{2}^{m-1}\right)^{4}\right\},
\end{aligned}
$$

where $m \geq 1$, then $\left|\nabla \Omega\left(\xi_{1}, \xi_{2}\right)\right|^{2}$ is an elliptic polynomial. Hence Theorem 3.4 applies.

3. Let $n \geq 2$ and $\Omega_{1}(\xi)=|\xi|^{2 m} \xi_{n}, \Omega_{2}(\xi)=|\xi|^{2 m} \sum_{i=1}^{n} \xi_{i}$, where $m \geq 1$ is an integer. Then both $\left|\nabla \Omega_{1}\right|^{2}$ and $\left|\nabla \Omega_{2}\right|^{2}$ are elliptic polynomials and hence Theorem 3.4 applies for both phase functions.

4. Let $n \geq 2$ and $\Omega(\xi)=\left(\sum_{1}^{n-1} \xi_{i}^{2}\right)^{m} \xi_{n}$, where $m \geq 1$ is an integer. Then $|\nabla \Omega|^{2}$ is not elliptic, but if $m \leq n-1, \Omega$ satisfies the conditions of Theorem 3.4.

Proofs. 1. Let $\operatorname{deg} \Omega=k$, and denote the real and imaginary parts of the complex polynomial $z^{k}=\left(\xi_{1}+i \xi_{2}\right)^{k}$ by $P_{k}\left(\xi_{1}, \xi_{2}\right)$ and $Q_{k}\left(\xi_{1}, \xi_{2}\right)$ respectively. Then there exist two real constants $A_{k}$ and $B_{k}$, not both equal to 0 , such that $\Omega\left(\xi_{1}, \xi_{2}\right)=$ $A_{k} P_{k}\left(\xi_{1}, \xi_{2}\right)+B_{k} Q_{k}\left(\xi_{1}, \xi_{2}\right)$ (cf. [25, Ch. 4]). Hence from the Cauchy-Riemann equations,

$$
\frac{\partial \Omega}{\partial \xi_{1}}=A_{k} \frac{\partial P_{k}}{\partial \xi_{1}}+B_{k} \frac{\partial Q_{k}}{\partial \xi_{1}}=A_{k} \frac{\partial P_{k}}{\partial \xi_{1}}-B_{k} \frac{\partial P_{k}}{\partial \xi_{2}},
$$

and

$$
\frac{\partial \Omega}{\partial \xi_{2}}=A_{k} \frac{\partial P_{k}}{\partial \xi_{2}}+B_{k} \frac{\partial Q_{k}}{\partial \xi_{2}}=A_{k} \frac{\partial P_{k}}{\partial \xi_{2}}+B_{k} \frac{\partial P_{k}}{\partial \xi_{1}}
$$


it follows that

$$
\begin{aligned}
& \left|\nabla \Omega\left(\xi_{1}, \xi_{2}\right)\right|^{2}=\left(\frac{\partial \Omega}{\partial \xi_{1}}\right)^{2}+\left(\frac{\partial \Omega}{\partial \xi_{2}}\right)^{2} \\
& =\left(A_{k}^{2}+B_{k}^{2}\right)\left\{\left(\frac{\partial P_{k}}{\partial \xi_{1}}\right)^{2}+\left(\frac{\partial P_{k}}{\partial \xi_{2}}\right)^{2}\right\}=\left(A_{k}^{2}+B_{k}^{2}\right)\left|\frac{d}{d z}\left(z^{k}\right)\right|^{2} \\
& =k^{2}\left(A_{k}^{2}+B_{k}^{2}\right)\left(\xi_{1}^{2}+\xi_{2}^{2}\right)^{k-1} .
\end{aligned}
$$

That is, $|\nabla \Omega|^{2}$ is an elliptic polynomial and therefore the conclusion follows.

2. From the homogeneity of $\Omega$, it suffices to show that $|\nabla \Omega(\xi)|^{2}$ has no zeros on the unit circle in $\mathbb{R}^{2}$. Suppose to the contrary that $|\nabla \Omega(\xi)|^{2}$ has zeroes on the unit circle in $\mathbb{R}^{2}$, then for some $\xi_{1}, \xi_{2}, \xi_{1}^{2}+\xi_{2}^{2}=1$, and $|\nabla \Omega(\xi)|=0$. But since

$$
\frac{\partial \Omega}{\partial \xi_{1}}=\left(\xi_{1}^{2}+\xi_{2}^{2}\right)^{-2}\left\{4 m \xi_{1}^{4 m-1}\left(\xi_{1}^{2}+\xi_{2}^{2}\right)-2 \xi_{1}\left(\xi_{1}^{4 m}-\xi_{2}^{4 m}\right)\right\},
$$

and

$$
\frac{\partial \Omega}{\partial \xi_{2}}=\left(\xi_{1}^{2}+\xi_{2}^{2}\right)^{-2}\left\{-4 m \xi_{2}^{4 m-1}\left(\xi_{1}^{2}+\xi_{2}^{2}\right)-2 \xi_{2}\left(\xi_{1}^{4 m}-\xi_{2}^{4 m}\right)\right\}
$$

it follows that $4 m \xi_{1}^{4 m-1}-2 \xi_{1}\left(\xi_{1}^{4 m}-\xi_{2}^{4 m}\right)=0$, and $-4 m \xi_{2}^{4 m-1}-2 \xi_{2}\left(\xi_{1}^{4 m}-\xi_{2}^{4 m}\right)=0$. Since $\xi_{1} \neq 0$, and $\xi_{2} \neq 0$, multiply the first equation by $\xi_{2}$ and the second one by $\xi_{1}$, to obtain $4 m \xi_{1}^{4 m-1} \xi_{2}=2 \xi_{1} \xi_{2}\left(\xi_{1}^{4 m}-\xi_{2}^{4 m}\right)=-4 m \xi_{2}^{4 m-1} \xi_{1}$, or equivalently that $\left(\xi_{1} / \xi_{2}\right)^{4 m-2}=-1$. This is impossible and hence $\left|\nabla \Omega\left(\xi_{1}, \xi_{2}\right)\right|^{2}$ is an elliptic polynomial.

3. We prove the ellipticity of $\left|\nabla \Omega_{2}\right|^{2}$ only since the proof for $\left|\nabla \Omega_{1}\right|^{2}$ is completely analogous. For this it suffices to show that $\left|\nabla \Omega_{2}\right|^{2}$ has no zeros on the unit sphere $S^{n-1}$ in $\mathbb{R}^{n}$. Assume to the contrary that for some $\xi \in S^{n-1},|\nabla \Omega(\xi)|=0$. Since

$$
\frac{\partial \Omega_{2}}{\partial \xi_{k}}=2 m \xi_{k}|\xi|^{2(m-1)} \sum_{1}^{n} \xi_{i}+|\xi|^{2 m}=|\xi|^{2(m-1)}\left(2 m \xi_{k} \sum_{1}^{n} \xi_{i}+|\xi|^{2}\right)
$$

it follows that $2 m \xi_{k} \sum_{1}^{n} \xi_{i}+1=0,1 \leq k \leq n$, and therefore $\xi_{k}=-\left(2 m \sum_{i=1}^{n} \xi_{i}\right)^{-1}$, that is, $\xi_{1}=\xi_{2}=\cdots=\xi_{n}$. Hence

$$
0=2 m \xi_{k} \sum_{1}^{n} \xi_{i}+1=2 m \xi_{1}\left(n \xi_{1}\right)+1=2 m n \xi_{1}^{2}+1 \geq 1
$$

a contradiction. Thus $\left|\nabla \Omega_{2}\right|^{2}$ is an elliptic polynomial.

4. Clearly

$$
\frac{\partial \Omega}{\partial \xi_{k}}=m\left(\sum_{1}^{n-1} \xi_{i}^{2}\right)^{m-1} 2 \xi_{k} \xi_{n}, \quad 1 \leq k \leq n-1, \quad \frac{\partial \Omega}{\partial \xi_{n}}=\left(\sum_{1}^{n-1} \xi_{i}^{2}\right)^{m} .
$$


Hence

$$
\begin{aligned}
|\nabla \Omega(\xi)|^{2}= & \sum_{k=1}^{n-1}\left\{\left(2 m\left(\xi_{1}^{2}+\xi_{2}^{2}+\cdots+\xi_{n-1}^{2}\right)^{m-1} \xi_{k} \xi_{n}\right\}^{2}\right. \\
& +\left\{\xi_{1}^{2}+\xi_{2}^{2}+\cdots+\xi_{n-1}^{2}\right\}^{2 m} \\
= & \left(\xi_{1}^{2}+\cdots+\xi_{n-1}^{2}\right)^{2 m-2}\left\{4 m^{2} \xi_{n}^{2} \sum_{k=1}^{n-1} \xi_{k}^{2}+\left(\xi_{1}^{2}+\cdots+\xi_{n-1}^{2}\right)^{2}\right\} \\
= & \left(\xi_{1}^{2}+\cdots+\xi_{n-1}^{2}\right)^{2 m-1}\left\{4 m^{2} \xi_{n}^{2}+\xi_{1}^{2}+\cdots+\xi_{n-1}^{2}\right\} \\
= & \left\{\xi_{1}^{2}+\xi_{2}^{2}+\cdots+\xi_{n-1}^{2}\right\}^{(2 m-1)}\left\{|\xi|^{2}+\left(4 m^{2}-1\right) \xi_{n}^{2}\right\} .
\end{aligned}
$$

Assuming that $|\xi|=1$ and $|\nabla \Omega(\xi)|^{2}=0$, that is,

$$
\left\{\xi_{1}^{2}+\xi_{2}^{2}+\xi_{3}^{2}+\cdots+\xi_{n-1}^{2}\right\}^{2 m-1}\left\{1+\left(4 m^{2}-1\right) \xi_{n}^{2}\right\}=0,
$$

which implies $\xi_{1}^{2}+\xi_{2}^{2}+\cdots+\xi_{n-1}^{2}=0$ and therefore $\xi_{1}=\xi_{2}=\xi_{3}=\cdots=\xi_{n-1}=0$ and $\xi_{n}= \pm 1$. Hence $|\nabla \Omega|$ has two zeros, on the unit sphere $S^{n-1}$, say

$$
\xi_{S}=(0, \ldots, 0,-1), \quad \xi_{N}=(0, \ldots, 0,+1) .
$$

To show that $\xi_{N}$ is a regular zero of order $2 m-1$, let the polar coordinates on $S^{n-1}$ be defined by

$$
\begin{aligned}
& \xi_{n}=\cos \theta_{1}, \\
& \xi_{n-1}=\sin \theta_{1} \cos \theta_{2}, \\
& \cdots, \\
& \xi_{2}=\sin \theta_{1} \sin \theta_{2} \cdots \sin \theta_{n-2} \cos \theta_{n-1}, \\
& \xi_{1}=\sin \theta_{1} \sin \theta_{2} \sin \theta_{3} \cdots \sin \theta_{n-2} \sin \theta_{n-1},
\end{aligned}
$$

where $\theta_{1}$ is the angle between the position vector $\xi=\left(\xi_{1}, \xi_{2}, \ldots, \xi_{n}\right)$ and the positive $\xi_{n}$ coordinate axis. By (4.1) one obtains on $S^{n-1}$

$$
\begin{aligned}
|\nabla \Omega(\xi)| & =\left\{\sum_{1}^{n-1} \xi_{i}^{2}\right\}^{m-1 / 2}\left\{1+\left(4 m^{2}-1\right)\left(\cos \theta_{1}\right)^{2}\right\}^{1 / 2} \\
& =\left[1-\xi_{n}^{2}\right]^{m-1 / 2}\left\{1+\left(4 m^{2}-1\right)\left(\cos \theta_{1}\right)^{2}\right\}^{1 / 2} \\
& =\left(\sin \theta_{1}\right)^{2 m-1}\left\{1+\left(4 m^{2}-1\right)\left(\cos \theta_{1}\right)^{2}\right\}^{1 / 2} \\
& \cong C \theta_{1}^{2 m-1}, \quad \text { as } \theta_{1} \rightarrow 0^{+},
\end{aligned}
$$

and hence $\xi_{N}$ is a regular zero of $|\nabla \Omega|$ of order $2 m-1$. The proof that $\xi_{S}$ is a regular zero of order $2 m-1$ follows by symmetry. In order that Theorem 3.4 applies, the order of the regular zeros should not be larger than $\left(1-\frac{1}{n}\right)$ times the order of $\Omega$. That is, $2 m-1 \leq(1-1 / n)(2 m+1)$ or $m \leq n-1$, which was assumed.

Remark 4.1. If $n=2$, it can be shown that for a real homogeneous polynomial $\Omega$, the zeros of $|\nabla \Omega|$ are always of regular type. Hence if the zeros satisfy the conditions of Theorem 3.4, the estimate (3.2) holds. It would be interesting to know whether (3.2) holds without the regularity conditions imposed on the zeros in Theorem 3.4.

Finally for the phase function $\Omega(\xi)=|\xi|^{d}, d>0$, sharp estimates for the global maximal operator $S_{d}^{* *}$ are given. 
In [4] A. Carbery proved for the local maximal operator $S_{d}^{*}$ :

$$
\left(\int_{\mathbb{R}^{n}}\left|\left(S_{d}^{*} f\right)(x)\right|^{2} d x\right)^{1 / 2} \leq C\|f\|_{H_{s}}
$$

if $s>d / 2, d>0$. Whether the index $d / 2$ is sharp seems not to be known. However, if a power weight on the range space of the operator is introduced, then the next theorem shows that a sharp estimate can be given. In fact such a result holds even for the global maximal operator $S_{d}^{* *}$.

Theorem 4.1. Let $n \geq 2,0<d \leq a<n, a>1$, and $f \in \mathcal{S}\left(\mathbb{R}^{n}\right)$, then

$$
\left(\int_{\mathbb{R}^{n}}\left|\left(S_{d}^{* *} f\right)(x)\right|^{2} \frac{d x}{|x|^{a}}\right)^{1 / 2} \leq C\|f\|_{H_{s}}
$$

holds for $s>a / 2$ and fails for $s<a / 2$.

Proof. Let $\varphi(t)=t^{d}, d>0$. With $\Omega(\xi)=\varphi(|\xi|)$, the conditions of Theorem 3.1 are satisfied with $0<a-1<n-1$. Hence for $\varepsilon>0$, there exists a constant $C>0$ independent of $f \in \mathcal{S}\left(\mathbb{R}^{n}\right)$ such that

$$
\begin{aligned}
& \left(\int_{\mathbb{R}^{n}}\left|\left(S_{d}^{* *} f\right)(x)\right|^{2} \frac{d x}{|x|^{a}}\right)^{1 / 2} \\
& \quad \leq C\left(\int_{\mathbb{R}^{n}} \frac{\sqrt{1+|\xi|^{2 d}}}{\left(d|\xi|^{d^{d}}\right)}|\xi|^{a-1}\left(1+|\xi|^{2}\right)^{\varepsilon}|\hat{f}(\xi)|^{2} d \xi\right)^{1 / 2} \\
& \quad \leq C\left(\int_{\mathbb{R}^{n}}\left(1+|\xi|^{2}\right)^{d / 2}|\xi|^{a-d}\left(1+|\xi|^{2}\right)^{\varepsilon}|\hat{f}(\xi)|^{2} d \xi\right)^{1 / 2} \\
& \quad \leq C\left(\int_{\mathbb{R}^{n}}\left(1+|\xi|^{2}\right)^{a / 2}\left(1+|\xi|^{2}\right)^{\varepsilon}|\hat{f}(\xi)|^{2} d \xi\right)^{1 / 2}=C\|f\|_{H_{a / 2+\varepsilon}} .
\end{aligned}
$$

This proves the first part of the theorem.

The failure of (4.2) for $s<a / 2$ follows from Lemma 2.1 as before (cf. Theorem $3.3)$.

Note that with $1<a=d<n,(4.2)$ has the form

$$
\left(\int_{\mathbb{R}^{n}}\left|\left(S_{d}^{* *} f\right)(x)\right|^{2} \frac{d x}{|x|^{d}}\right)^{1 / 2} \leq C\|f\|_{H_{s}}
$$

where $s>d / 2$. This may be viewed as the sharp estimate for $S_{d}^{* *}$ corresponding to Carbery's result.

From (4.2) and the obvious inequality (cf. proof of Proposition 3.2)

$$
\sup _{x \in \mathbb{R}^{n}}\left|\left(S_{d}^{* *} f\right)(x)\right| \leq C\|f\|_{H_{s}}
$$

for $s>n / 2$, one obtains on interpolation:

Corollary 4.1. Let $n \geq 2,0<d \leq a<n, a>1, p>2$, then the estimate

$$
\left(\int_{\mathbb{R}^{n}}\left|\left(S_{d}^{* *} f\right)(x)\right|^{p} \frac{d x}{|x|^{a}}\right)^{1 / p} \leq C\|f\|_{H_{s}}
$$

holds for $s>n(1 / 2-1 / p)+a / p$ and fails for $s<n(1 / 2-1 / p)+a / p$. 
The failure of (4.3) for $s<n(1 / 2-1 / p)+a / p$ follows again from Lemma 2.1.

If $d=2$, interpolation between an estimate of Kenig, Ponce and Vega and (4.3) yields:

Corollary 4.2. Let $n \geq 3,0 \leq a<n, p \geq 4$, then

$$
\left(\int_{\mathbb{R}^{n}}\left|\left(S_{2}^{* *} f\right)(x)\right|^{p} \frac{d x}{|x|^{a}}\right)^{1 / p} \leq C\|f\|_{H_{s}}
$$

holds for $s>n(1 / 2-1 / p)+a / p$ and fails for $s<n(1 / 2-1 / p)+a / p$.

Proof. It is known (cf. [13, p. 14]) that

$$
\left(\int_{\mathbb{R}^{n}}\left|\left(S_{2}^{* *} f\right)(x)\right|^{4} d x\right)^{1 / 4} \leq C\|f\|_{H_{n / 4}}
$$

and by Corollary 4.1 with $p=4$

$$
\left(\int_{\mathbb{R}^{n}}\left|\left(S_{2}^{* *} f\right)(x)\right|^{4} \frac{d x}{|x|^{a}}\right)^{1 / 4} \leq\|f\|_{H_{(n+a) / 4+\varepsilon}},
$$

where $\varepsilon>0,2<a<n$. Interpolating between these estimates shows that

$$
\left(\int_{\mathbb{R}^{n}}\left|\left(S_{2}^{* *} f\right)(x)\right|^{4} \frac{d x}{|x|^{a}}\right)^{1 / 4} \leq C\|f\|_{H_{(a+n) / 4+\varepsilon}},
$$

where $0 \leq a<n$, and $\varepsilon>0$. Finally interpolating between (4.4) and

$$
\left\|\left(S_{2}^{* *} f\right)\right\|_{L^{\infty}\left(\mathbb{R}^{n},|x|^{-a} d x\right)} \leq\|f\|_{H_{n / 2+\varepsilon}},
$$

yields the first part of Corollary 4.2.

The negative result can be proved via the same method used in the proof of Theorem 3.3.

Note that if $\Delta$ is the Laplacian, then by Plancherel's theorem

$$
\left(\int_{\mathbb{R}^{n}}\left|(1-\Delta)^{s / 2} f(x)\right|^{2} d x\right)^{1 / 2}=\left(\int_{\mathbb{R}^{n}}\left(1+|\xi|^{2}\right)^{s}|\hat{f}(\xi)|^{2} d \xi\right)^{1 / 2} .
$$

If $1<p<\infty$, define $H_{s}^{p}$ by

$$
f \in H_{s}^{p}, \quad \text { if } \quad\|f\|_{H_{s}^{p}}=\left(\int_{\mathbb{R}^{n}}\left|(1-\Delta)^{s / 2} f(x)\right|^{p} d x\right)^{1 / p}<+\infty .
$$

Then $H_{s}^{2}=H_{s}$.

Proposition 4.2. Let $n \geq 2,1<a<n$, and $f \in \mathcal{S}\left(\mathbb{R}^{n}\right)$.

(i) If $a<d<n+a, p=2 n /(n+d-a)$ and $s>d / 2$, then

$$
\left(\int_{\mathbb{R}^{n}}\left|\left(S_{d}^{* *} f\right)(x)\right|^{2} \frac{d x}{|x|^{a}}\right)^{1 / 2} \leq C\|f\|_{H_{s}^{p}}
$$

holds.

(ii) If $0<d<n, p=2 n /(n+d)$ and $s>(a+d) / 2$, then

$$
\left(\int_{\mathbb{R}^{n}}\left|\left(S_{d}^{* *} f\right)(x)\right|^{2} \frac{d x}{|x|^{a}}\right)^{1 / 2} \leq C\|f\|_{H_{s}^{p}}
$$

holds. 
Proof. (i) From Theorem 3.1 it follows that for $\varepsilon>0$,

$$
\begin{aligned}
&\left(\int_{\mathbb{R}^{n}}\right.\left.\left|\left(S_{d}^{* *} f\right)(x)\right|^{2} \frac{1}{|x|^{a}} d x\right)^{1 / 2} \\
& \quad \leq C\left(\int_{\mathbb{R}^{n}}\left(1+|\xi|^{2}\right)^{d / 2}|\xi|^{a-d}\left(1+|\xi|^{2}\right)^{\varepsilon}|\hat{f}(\xi)|^{2} d \xi\right)^{1 / 2} \\
& \quad= C\left(\left.\left.\int_{\mathbb{R}^{n}}|| \xi\right|^{-(d-a) / 2}\left(1+|\xi|^{2}\right)^{(d / 2+\varepsilon) / 2} \hat{f}(\xi)\right|^{2} d \xi\right)^{1 / 2} \\
& \quad=C\left(\int_{\mathbb{R}^{n}}\left|\mathcal{F}\left(I_{(d-a) / 2}(1-\Delta)^{(d / 2+\varepsilon) / 2} f\right)(x)\right|^{2} d x\right)^{1 / 2},
\end{aligned}
$$

where $\mathcal{F}$ denotes the Fourier transformation and $I_{(d-a) / 2},(1-\Delta)^{[(d / 2+\varepsilon] / 2}$ denote the Riesz potential operator of order $(d-a) / 2$ and the Bessel potential operator of order $-[d / 2+\varepsilon]$, respectively. By Plancherel's theorem and the Hardy-LittlewoodSobolov inequality, the last integral is equal to

$$
\begin{aligned}
& \left(\int_{\mathbb{R}^{n}}\left|\left(I_{(d-a) / 2}(1-\Delta)^{(d / 2+\varepsilon) / 2} f\right)(x)\right|^{2} d x\right)^{1 / 2} \\
& \quad \leq C\left(\int_{\mathbb{R}^{n}}\left|(1-\Delta)^{(d / 2+\varepsilon) / 2} f(x)\right|^{p} d x\right)^{1 / p}=C\|f\|_{H_{(d / 2+\varepsilon)}^{p}},
\end{aligned}
$$

where $1-(d-a) /(2 n)=1-(1 / p-1 / 2), 1<p<2$, that is, $p=2 n /(n+d-a), 0<$ $d-a<n$. The proof for (i) is thus completed.

For (ii), again apply Theorem 3.1 to obtain

$$
\begin{aligned}
& \left(\int_{\mathbb{R}^{n}}\left|\left(S_{d}^{* *} f\right)(x)\right|^{2} \frac{d x}{|x|^{a}}\right)^{1 / 2} \\
& \quad \leq C\left(\int_{\mathbb{R}^{n}} \frac{\sqrt{1+|\xi|^{2 d}}}{\left(d|\xi|^{d-1}\right)}|\xi|^{a-1}\left(1+|\xi|^{2}\right)^{\varepsilon}|\hat{f}(\xi)|^{2} d \xi\right)^{1 / 2} \\
& \leq C\left(\int_{\mathbb{R}^{n}}|\xi|^{-d}\left(1+|\xi|^{2}\right)^{(d+a) / 2+\varepsilon}|\hat{f}(\xi)|^{2} d \xi\right)^{1 / 2} \\
& \quad=C\left(\int_{\mathbb{R}^{n}}\left|\mathcal{F}\left(I_{d / 2}(1-\Delta)^{[(d+a) / 2+\varepsilon] / 2} f\right)(x)\right|^{2} d x\right)^{1 / 2} \\
& \leq C\left(\int_{\mathbb{R}^{n}}\left|(1-\Delta)^{[(d+a) / 2+\varepsilon] / 2} f(x)\right|^{p} d x\right)^{1 / p}=C\|f\|_{H_{(d+a) / 2+\varepsilon}^{p}}
\end{aligned}
$$

Here $\mathcal{F}, I_{d / 2}$, and $(1-\Delta)^{[(d+a) / 2+\varepsilon] / 2}$ are the Fourier transform, the Riesz potential of order $d / 2$, and Bessel potential of order $-[(d+a) / 2+\varepsilon] / 2$ respectively. Applying first Plancherel's theorem and then the Hardy-Littlewood-Sobolev theorem, the last inequality holds if

$$
1-\frac{d}{2 n}=1-\left(\frac{1}{p}-\frac{1}{2}\right), \quad \text { or } \quad p=2 n /(n+d)
$$

This proves the proposition. 


\section{REFERENCES}

1. J. J. Benedetto, H. P. Heinig, and R. Johnson, Weighted Hardy spaces and the Laplace transform. II, Math. Nachr. 132 (1987), 29-55. MR 88m:44007

2. J. Bergh and J. Löfström, Interpolation Spaces, An Introduction, Springer, New York, 1976. MR 58:2349

3. J. Bourgain, A remark on Schrödinger operators, Israel J. Math. 77 (1992), 1-16. MR 93k:35071

4. A. Carbery, Radial Fourier multipliers and associated maximal functions, North-Holland Math. Studies, vol. III, North-Holland, 1985, 49-55. MR 87i:42029

5. L. Carleson, Some analytical problems related to statistical mechanics, Euclidean Harmonic Analysis, Lecture Notes in Math. 779 (1979), 5-45. MR 82j:82005

6. P. Constantin and J. C. Saut, Local smoothing properties of dispersive equations, J. Amer. Math. Soc. 1 (1989), 413-446. MR 89d:35150

7. M. Cowling, Pointwise behavior of solutions to Schrödinger equations, Harmonic Analysis, Lecture Notes in Math. 992 (1983), 83-90. MR 85c:34029

8. B. E. J. Dahlberg and C. E. Kenig, A note on almost everywhere behavior of solutions to the Schrödinger equation, Harmonic Analysis, Lecture Notes in Math., 908 (1982), 205-209. MR 83f: 35023

9. H. P. Heinig, Weighted norm inequalities for classes of operators, Indiana Univ. Math. J. (4) 33 (1984), 573-582. MR 86c: 42016

10. L. Hörmander, The Analysis of Linear Partial Differential Operators, Vol. 1, second ed., Springer, 1983. MR 85g:35002a

11. C. E. Kenig and A. Ruiz, A strong type $(2,2)$ estimate for a maximal operator associated to the Schrödinger equation, Trans. Amer. Math. Soc. 280 (1983), 239-246. MR 85c:42010

12. C. E. Kenig, D. Ponce, and L. Vega, Oscillatory integrals and regularity of dispersive equations, Indiana Univ. Math. J. 40 (1991), 33-69. MR 92d:35081

13. $ـ$ On the IVP for the non-linear Schrödinger equations, Contemp. Math., 189, Amer. Math. Soc., Providence, R.I., 1995. MR 96e:35071

14. $ـ$ Small solutions to non-linear Schrödinger equations, Ann. Inst. H. Poincaré Anal. Non Linéaire 10 (1993), 255-288. MR 94h:35238

15. W Well-posedness and scattering results for the generalized Korteweg-de Vries equation via the contractions principle, Comm. Pure Appl. Math. 46 (1993), 527-620. MR 94h:35229

16. E. Prestini, Radial functions and regularity of solutions to the Schrödinger equation, Monatsh. Math. 109 (1990), 135-143. MR 91j:35035

17. W. Rudin, Principles of Mathematical Analysis, third ed., McGraw-Hill, 1976. MR 52:5893

18. H.-J. Schmeisser and H. Triebel, Topics in Fourier Analysis and Function Spaces, Wiley, New York, 1985. MR 88k:42015b

19. P. Sjölin, Regularity of solutions to the Schrödinger equation, Duke Math. J. 55 (1987), 699715. MR 88j:35026

20. _ Radial functions and maximal estimates for solutions to the Schrödinger equation, J. Austral. Math. Soc. Ser. A. 59 (1995), 134-142. MR 96d:42032

21. Global maximal estimates for solutions to the Schrödinger equation, Studia Math. 110 (1994), 105-114. MR 95e:35052

22. _— $L^{p}$ maximal estimates for solutions to the Schrödinger equation, Private Communication, Aug. 1994.

23. C. Sogge, Fourier integrals in classical analysis, Cambridge Tracts in Mathematics, 105, Cambridge Univ. Press, 1993. MR 94c:35178

24. E. M. Stein, Singular integrals and differentiability properties of functions, Princeton Univ. Press, Princeton, N.J., 1970. MR 44:7280

25. E. M. Stein and G. Weiss, Introduction to Fourier analysis on Euclidean spaces, Princeton Univ. Press, Princeton, N.J., 1971. MR 46:4102

26. - Interpolation of operators with change of measures, Trans. Amer. Math. Soc. 87 (1958), 159-172. MR 19:1184d

27. H. Triebel, Theory of Function Spaces, Monographs in Math., 78, Birkhäuser, 1983. MR $\mathbf{8 6 j}: 46026$

28. L. Vega, Schrödinger equations: pointwise convergence to the initial data, Proc. Amer. Math. Soc. 102 (1988), 874-878. MR 89d:35046 
29. B. Walther, Maximal estimates of oscillatory integrals with concave phase, Preprint, 25 (1994), Math. Dept., Uppsala Univ., Sweden.

30. Sichun Wang, On the maximal operator associated with the Schrödinger equation, Studia Math. 122 (1997), 167-182. MR 98f:42019

31. Silei Wang, On the weighted estimate of the solution associated with the Schrödinger equation, Proc. Amer. Math. Soc. 113 (1991), 87-92. MR 91k:35066

32. G. N. Watson, A Treatise on the Theory of Bessel Functions, Cambridge Univ. Press, 1922.

Department of Mathematics and Statistics, McMaster University, Hamilton, OnTARIO, L8S 4K1, CANADA

E-mail address: heinig@mcmail.cis.mcmaster.ca

E-mail address: wangs@icarus.math.mcmaster.ca 